\title{
A New Sinusoidal Shear Deformation Theory for Static Bending Analysis of Functionally Graded Plates Resting on Winkler-Pasternak Foundations
}

\author{
Pham Minh Phuc $i$ and Vu Nguyen Thanh \\ Faculty of Basic Sciences, University of Transport and Communications, 03 Cau Giay Street-Dong Da, Hanoi, Vietnam \\ Correspondence should be addressed to Pham Minh Phuc; bmclt@utc.edu.vn
}

Received 28 November 2020; Revised 4 April 2021; Accepted 17 April 2021; Published 3 May 2021

Academic Editor: Chaohui Wang

Copyright (c) 2021 Pham Minh Phuc and Vu Nguyen Thanh. This is an open access article distributed under the Creative Commons Attribution License, which permits unrestricted use, distribution, and reproduction in any medium, provided the original work is properly cited.

\begin{abstract}
In this article, a new sinusoidal shear deformation theory was developed for static bending analysis of functionally graded plates resting on elastic foundations. The proposed theory used an undefined integral term to reduce the number of the unknown to four without any shear correction factors. The high accuracy and efficiency of the proposed theory were proved thanks to the comparisons of the present results with other available solutions. And then, the proposed theory was successfully applied to investigate the bending behavior of the functionally graded plates resting on Winkler-Pasternak foundations. The governing equations of motion were established by using Hamilton's principle, and the Navier's solution technique was employed to solve these equations. The effects of some factors of the geometrics, the materials properties, and the elastic foundation parameters on the bending behaviors of the FGM plates were investigated intensely. Also, some novel results and special phenomenon were carried out.
\end{abstract}

\section{Introduction}

Functionally graded materials (FGMs) are made from a mixture of two or more ingredients together. In such type of material, the material properties vary continuously through the thickness of the structures. Because of their exotic properties, FGMs are widely applied in many fields of engineering and industry [1-6], for example, aeronautics, nuclear engineering, advanced civil engineering, and so on. More details of the application and investigation of FGMs can be read from a state-of-the-art review by Swaminathan et al. [7]. So many scientists focused on investigating the mechanical behavior, thermal response, mechanical-electrical behavior of FGM structures such as beams, plates, and shells, especially FGM plates and shells. So, finding a simple, efficient, and suitable shear deformation to analyze these structures is one of the biggest challenges of researchers. Many plate theories were developed and applied successfully in the past, such as classical plate theory (CPT), Mindlin plate theory or first-order shear deformation theories (FSDTs), higher-order shear deformation plate theories (HSDTs), quasi-3D deformation theories, and their variations.

Firstly, Liessa [8] researched the free vibration of rectangular plates using CPT. Javaheri et al. [9] applied CPT to investigate the buckling behavior of FGM plates subjected to in-plane compressive load. Mohammadi et al. [10] used Levy type solution based on CPT to study buckling of rectangular FGM plates. A deep investigation of the effects of some parameters on the vibration and stability of FGM plates was carried out by $\mathrm{Hu}$ et al. [11]. One of the most disadvantages of the CPT is that the transverse shear strains are neglected, so CPT is just suitable to investigate thin and very thin plates and cannot be applied to study thick and very thick plates.

To address the disadvantage of CPT, Reissner-Mindlin plate theory and FSDTs were developed to investigate moderate thick plates. In such type of plate theory, the transverse shear strains are considered. Croce et al. [12] 
applied Reissner-Mindlin plate theory incorporated with finite element method (FEM) to investigate the mechanical behavior of FGM plates in a thermal environment. The buckling of the skew FGM plate subjected to mechanical load was investigated by Ganapathi and his coworkers [13]. Kim et al. [14] studied geometrically nonlinear behavior of FGM plates and shells using FSDT and a four-node quasiconforming shell element. Hosseini-Hashemi and his coworkers employed FSDT [15] and Reissner-Mindlin [16] to study the free vibration of rectangular FGM plates. Nguyen et al. [17] analyzed the mechanical behavior of FGM plates using FSDT. Shimpi et al. [18] developed a new FSDT to investigate the bending behavior of plates. Thai et al. [19] developed a simple FSDT with only four unknown displacement functions to examine the bending and free vibration of FGM plates. A new FSDT was developed by Thai et al.[20] to analyze FGM plates. Nguyen et al. [21] established a refined simple FSDT in which the transverse shear stresses distribution is parabolical through the thickness of the plate, so it does not need any correction factors. Yu et al. [22] used a simple FSDT and isogeometric analysis to investigate the nonlinear bending of FGM plates. Jalaei et al. [23] applied FSDT in combination with nonlocal elasticity to analyze the dynamic instability of FGM nanobeams with porosity. Vu et al. [24] developed a new FSDT to investigate static bending and vibration of two-layer plates. Senjanović et al. [25] modified the Mindlin plate theory to investigate plates with a shear locking-free rectangular plate element. Although FSDT considered the transverse shear stresses, it still needs a correction factor to avoid the shear locking phenomenon. Moreover, the shear stresses cannot be predicted correctly and are only applied to thin and moderate plates.

To overcome these drawbacks of FSDTs, many HSDTs have been developed. In comparison with CPT and FSDTs, HSDTs have many significant benefits, such as that the transverse shear stresses are parabolically distributed through the thickness and satisfy the shear-free conditions on two surfaces of the plates, so they do not need any shear correction factors. In addition, HSDT can predict very well deflections and stresses of thin to moderate and thick plates. Javaheri et al. [26] used HSDT to investigate thermal bucking of FGM plates. Yang et al. [27] studied the dynamic stability of FGM plates using HSDT. Bodaghi et al. [28] used HSDT and Levy type solution to study buckling of thick FGM plates. Ferreira et al. [29] investigated the static bending behavior of FGM plates using third-order shear deformation theory (TSDT) and the meshless method. Tran et al. [30] used HSDT in combination with isogeometric analysis to study the mechanical behavior of FGM plates. A generalized shear deformation theory (GSDT) was developed by Zenkour [31] and was applied to analyze the bending behavior of FGM plates. Shimpi [32] developed two refined plate theories (RPT) and applied them to investigate static bending of plates. Van et al. [33] modified the RPT to investigate static bending of FGM plates. A simple HSDT and a sinusoidal shear deformation theory (SSDT) were developed by Thai et al. $[34,35]$ to analyze static bending, free vibration, and buckling of FGM plates. Vinh et al. [36] developed a new hyperbolic shear deformation theory in conjunction with
FEM to analyze FGM Sandwich plate with porosity. Touratier [37] developed a new sinusoidal HSDT to analyze composite plates. Akgoz et al. [38] applied a SSDT in combination with strain gradient elasticity theory to analyze static bending and free vibration of microplates. Mechab et al. [39] developed a new four-variable refined plate theory with a new function to study static bending and dynamic response of FGM plates. Meiche et al. [40] developed a new hyperbolic shear deformation theory to analyze buckling and free vibration of FG Sandwich plates. Menasria et al. [41] established a new simple HSDT to study the thermal stability of FG Sandwich plates. Pandya et al. [42] applied HSDT and FEM to study the flexure of Sandwich plates. Talha et al. [43] investigated static bending behavior as well as free vibration of FGM plates. Do et al. [44] analyzed the bidirection FGM plates using FEM and new TSDT, in which the authors showed that the materials have significant roles in the behavior of the FGM plates. Vinh et al. [45] and Hoa et al. [46] developed a single variable HSDT for static bending and free vibration analysis of FGM plates and FGM nanoplates. Zenkour et al. [47] developed a simple four unknown refined theories for the analysis of static bending of FGM plates. Nguyen et al. [48] developed a new inverse trigonometric shear deformation theory for the analysis of isotropic and FGM Sandwich plates.

Although HSDT and their variations have many benefits, the normal stress in $z$-direction is neglected, so they cannot predict exactly the behaviors of very thick plates. In recent years, many quasi-3D theories were developed, in which the deflection and normal stress in $z$-direction are considered. As a consequence, these types of plate theories are appropriate for the analysis of very thick plates. Qian et al. [49] used HSDT and normal deformation theory incorporated with meshless local Petrov-Galerkin method (MLPG) to investigate dynamic and static bending behavior of thick FGM plates. Gilhooley et al. [50] analyzed thick FGM plates using HSDT and normal deformation plate theory incorporated with MLPG and radial basis functions. Mantari et al. [51-54] developed a family of quasi-3D theories for analysis static bending, free vibration and buckling of FGM plates. Nguyen et al. [55] developed an efficient bean element based on quasi-3D theory to analyze the bending behavior of FGM beams. Zenkour et al. [56, 57] developed many quasi-3D theories for the analysis of isotropic and FGM Sandwich plates. Vinh [58] developed a hybrid quasi-3D theory for deflections, stress, and free vibration analysis of bi-FGM Sandwich plates resting on elastic foundation. Thai et al. [59] developed a simple quasi-3D sinusoidal shear deformation theory for FGM plates analysis. Neves et al. [60,61] established some efficient quasi-3D and applied to analyze static bending, free vibration, and buckling of isotropic and Sandwich FGM plates.

Analysis of beams, plates, and shells structures with elastic foundation support is an important problem in engineering. In comparison with the structures without elastic foundation support, the behavior of these structures resting on elastic foundation is completely different. Chakraverty et al. [62] studied free vibration of thin FGM plates resting on the Winkler foundation with general boundary conditions by Rayleigh-Ritz method. Mantari et al. [63] analyzed free vibration of composite plates resting on elastic 
foundation. Akavci [64] developed an efficient shear deformation to analyze free vibration of FGM thick plates resting on elastic foundation. Thai et al. [65] studied bending, buckling, and free vibration of thick FGM plates resting on elastic foundations using simple, refined theory. In other works of Thai et al. [66], a closed-form solution was developed to investigate the buckling of thick FGM plates. Baferani et al. [67] developed an accurate solution for free vibration analysis of FGM thick rectangular plates. Ameur et al. [68] developed a new trigonometric shear deformation theory to analyze the bending behavior of FGM plates resting on elastic foundations with the Winkler-Pasternak type model. Al Khateeb et al. [69] used a refined four-unknown plate theory to analyze advanced plates resting on elastic foundations in the hygrothermal environment. Attia et al. [70] employed a refined four variable plate theory for thermoelastic analysis of FGM plates supported by a variable elastic foundation. Avcar et al. [71] studied free vibration of FGM plates resting on elastic foundations with the Winkler-Pasternak type model. Benyoucef et al. [72] studied the bending behavior of FGM plates resting on the Winkler-Pasternak foundation. Han et al. [73] analyzed plates resting on the two-parameter foundation using the numerical differential quadrature method and the Reissner-Mindlin plate model. Gupta et al. [74] studied free vibration and bending response of FGM plates supported by Winkler-Pasternak foundations using nonpolynomial HSDT and normal shear deformation theory. Said et al. [75] developed a new simple hyperbolic shear deformation theory to analyze FGM plates resting on elastic foundations with the Winkler-Pasternak model. Zaoui et al. [76] developed new $2 \mathrm{D}$ and quasi-3D shear deformation theories for free vibration of FGM plates resting on elastic foundations.

This study aims to establish a novel sinusoidal shear deformation theory for bending behavior analysis of FGM plates supported by elastic foundations with the Winkler-Pasternak type model. This theory considered the parabolical distribution of the shear stresses and satisfied the free conditions of those on top and bottom surfaces of the plates. Hamilton's principle is applied to construct the governing equations of motion and the Navier's solution technique is used to solve these equations. The accuracy and efficiency of the proposed theory are proved thanks to several validation studies. Then the proposed theory is employed to investigate the flexure behavior of the FGM plates resting on Winkler-Pasternak type foundations. Several numerical investigations on the effects of some parameters are carried out and some new results and special phenomenon are illustrated.

\section{Theoretical Formulation}

\subsection{FGM Plates Resting on Winkler-Pasternak Foundations.} Figure 1 shows the model of FGM plates with the dimension of $a \times b$ and the thickness of $h$ lies on the elastic foundation. The material properties of the plate are assumed to vary continuously from bottom to top surfaces of the plates by a power-law function. In this study, the elastic foundation is modelled by the Winkler-Pasternak type that consists of two components which are a Winkler foundation with the stiffness of $k_{w}$ and a shear layer with the stiffness of $k_{s}$.

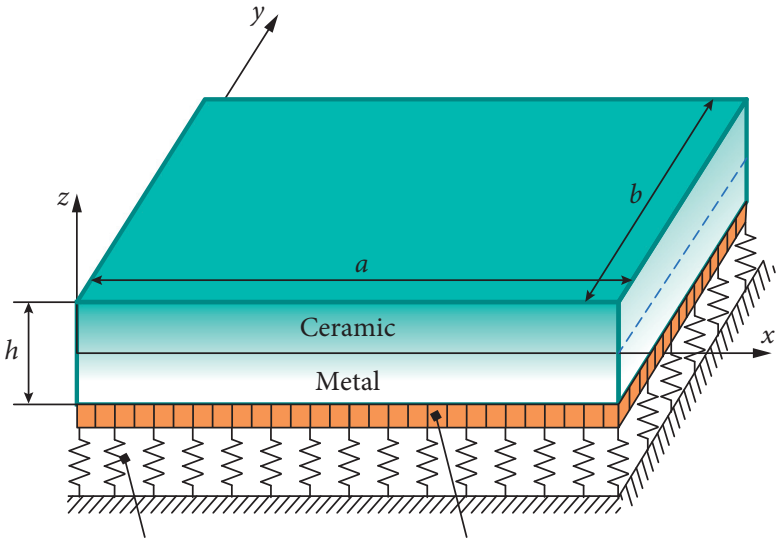

Winkler's layer $\left(k_{w}\right) \quad$ Shear layer $\left(k_{s}\right)$

FIGURE 1: The model of FGM plates resting on Winkler-Pasternak foundations.

The material properties of the plates are assumed to vary continuously through the thickness of the plates as powerlaw functions $[3,4]$.

$$
\begin{aligned}
& E(z)=E_{m}+\left(E_{c}-E_{m}\right) V_{f}, \\
& \nu(z)=v_{m}+\left(v_{c}-v_{m}\right) V_{f},
\end{aligned}
$$

where

$$
V_{f}=\left(\frac{1}{2}+\frac{z}{h}\right)^{p}
$$

where $E_{c}, E_{m}, v_{c}$ and $v_{m}$ are Young's modulus and Poisson's ratio of the ceramic and metal, respectively, $p$ is the powerlaw exponent, and $h$ is the thickness of the plates.

\subsection{New Sinusoidal Shear Deformation Theory}

2.2.1. Displacement Field. The displacement field at any point in the plates using new sinusoidal shear deformation theory is written by the following:

$$
\begin{aligned}
& u(x, y, z)=u(x, y)-z \frac{\partial w}{\partial x}+f(z)\left(\frac{\partial \theta}{\partial x}+\frac{\partial w}{\partial x}\right) \\
& v(x, y, z)=v(x, y)-z \frac{\partial w}{\partial y}+f(z)\left(\frac{\partial \theta}{\partial y}+\frac{\partial w}{\partial y}\right) \\
& w(x, y, z)=w(x, y) .
\end{aligned}
$$

By introducing two unknown derivative quantities $\partial \theta / \partial x$ and $\partial \theta / \partial y$, the proposed shear deformation theory consists of only four unknown displacement functions, it is similar to the simple FSDT of Thai et al. [19] and Ameur et al. [68]. The number of unknowns of the proposed theory is smaller than other HSDTs with five to eight unknowns, so the computation cost can be reduced. However, the transverse displacement is not separated into two parts as in simple FSDT or simple HSDT, so it is simpler and more efficient than other HSDTs. Moreover, other plate theories can be obtained easily by varying the functions $f(z)$. For example, the CPT 
can be taken by setting $f(z)=0$, the FSDT can be achieved by setting $f(z)=z$, and the HSDT of Reddy [3] can be achieved by setting $f(z)=z\left(1-4 z^{2} / 3 h^{2}\right)$. In this study, a new sinusoidal shear deformation theory is obtained by setting the following:

$$
f(z)=\frac{h \sqrt{15}}{3 \pi} \sin \left(\frac{\pi z}{h}\right) .
$$

The new sinusoidal shear deformation theory satisfies two conditions of the shear strains and stresses of the plates.
The first condition is that the distribution of the shear stresses through the thickness of the plates is parabolical. The second condition is that the shear strains and stresses equal to zeros at any points on two free surfaces of the plates. So, the proposed theory does not need any shear correction factors as in the FSDTs.

2.2.2. Constitutive Equations. The strains fields of the plate are written as follows:

$$
\begin{aligned}
\varepsilon_{x} & =\frac{\partial u}{\partial x}-z \frac{\partial^{2} w}{\partial x^{2}}+f(z)\left(\frac{\partial^{2} \theta}{\partial x^{2}}+\frac{\partial^{2} w}{\partial x^{2}}\right), \\
\varepsilon_{y} & =\frac{\partial v}{\partial y}-z \frac{\partial^{2} w}{\partial y^{2}}+f(z)\left(\frac{\partial^{2} \theta}{\partial y^{2}}+\frac{\partial^{2} w}{\partial y^{2}}\right), \\
\gamma_{x y} & =\frac{\partial u}{\partial y}+\frac{\partial v}{\partial x}-z\left(\frac{\partial^{2} w}{\partial x \partial y}+\frac{\partial^{2} w}{\partial y \partial x}\right)+2 f(z)\left(\frac{\partial^{2} \theta}{\partial y \partial x}+\frac{\partial^{2} w}{\partial x \partial y}\right), \\
\gamma_{x z} & =\frac{\partial w}{\partial x}-\frac{\partial w}{\partial x}+f^{\prime}(z)\left(\frac{\partial \theta}{\partial x}+\frac{\partial w}{\partial x}\right)=g(z)\left(\frac{\partial \theta}{\partial x}+\frac{\partial w}{\partial x}\right), \\
\gamma_{y z}= & \frac{\partial w}{\partial y}-\frac{\partial w}{\partial y}+f^{\prime}(z)\left(\frac{\partial \theta}{\partial y}+\frac{\partial w}{\partial y}\right)=g(z)\left(\frac{\partial \theta}{\partial y}+\frac{\partial w}{\partial y}\right),
\end{aligned}
$$

where

$$
g(z)=f^{\prime}(z)=\frac{\sqrt{15}}{3} \cos \left(\frac{\pi z}{h}\right) .
$$

From equations (5) and (6), it can be obvious that the free conditions of the shear strains and stresses on the top and bottom surfaces of the plates are satisfied automatically.

The strains fields of the plates can be written in compact form as follows:

$$
\begin{aligned}
& \boldsymbol{\varepsilon}=\left\{\begin{array}{c}
\varepsilon_{x} \\
\varepsilon_{y} \\
\gamma_{x y}
\end{array}\right\}=\boldsymbol{\varepsilon}^{0}+z \boldsymbol{\kappa}^{b}+f \boldsymbol{\kappa}^{s}, \\
& \boldsymbol{\gamma}=\left\{\begin{array}{l}
\gamma_{x z} \\
\gamma_{y z}
\end{array}\right\}=g \boldsymbol{\gamma}^{0},
\end{aligned}
$$

where

$$
\begin{aligned}
& \boldsymbol{\varepsilon}^{0}=\left\{\begin{array}{c}
\frac{\partial u}{\partial x} \\
\frac{\partial v}{\partial y} \\
\frac{\partial u}{\partial y}+\frac{\partial v}{\partial x}
\end{array}\right\}, \boldsymbol{\kappa}^{b}=-\left\{\begin{array}{c}
\frac{\partial^{2} w}{\partial x^{2}} \\
\frac{\partial^{2} w}{\partial y^{2}} \\
2 \frac{\partial^{2} w}{\partial x \partial y}
\end{array}\right\}, \boldsymbol{\kappa}^{s}=\left\{\begin{array}{c}
\frac{\partial^{2} \theta}{\partial x^{2}}+\frac{\partial^{2} w}{\partial x^{2}} \\
\frac{\partial^{2} \theta}{\partial y^{2}}+\frac{\partial^{2} w}{\partial y^{2}} \\
2\left(\frac{\partial^{2} \theta}{\partial x \partial y}+\frac{\partial^{2} w}{\partial x \partial y}\right)
\end{array}\right\}, \\
& \gamma^{0}=\left\{\begin{array}{l}
\frac{\partial \theta}{\partial x}+\frac{\partial w}{\partial x} \\
\frac{\partial \theta}{\partial y}+\frac{\partial w}{\partial y}
\end{array}\right\} .
\end{aligned}
$$


The constitutive equation of the plate is as follows [3]:

$$
\left\{\begin{array}{c}
\sigma_{x} \\
\sigma_{y} \\
\tau_{y z} \\
\tau_{x z} \\
\tau_{x y}
\end{array}\right\}=\left[\begin{array}{ccccc}
Q_{11} & Q_{12} & 0 & 0 & 0 \\
Q_{12} & Q_{22} & 0 & 0 & 0 \\
0 & 0 & Q_{44} & 0 & 0 \\
0 & 0 & 0 & Q_{55} & 0 \\
0 & 0 & 0 & 0 & Q_{66}
\end{array}\right]\left\{\begin{array}{c}
\varepsilon_{x} \\
\varepsilon_{y} \\
\gamma_{y z} \\
\gamma_{x z} \\
\gamma_{x y}
\end{array}\right\},
$$

where

$$
\begin{aligned}
& Q_{11}=Q_{22}=\frac{E(z)}{1-v^{2}(z)}, \\
& Q_{12}=\frac{\nu(z) E(z)}{1-v^{2}(z)}, \\
& Q_{66}=Q_{55}=Q_{44}=\frac{E(z)}{2(1+\nu(z))} .
\end{aligned}
$$

It can be written in short form as the following equation:

$$
\left\{\begin{array}{l}
\boldsymbol{\sigma} \\
\boldsymbol{\tau}
\end{array}\right\}=\left[\begin{array}{cc}
\mathbf{D}_{b} & \mathbf{0} \\
\mathbf{0} & \mathbf{D}_{s}
\end{array}\right]\left\{\begin{array}{l}
\boldsymbol{\varepsilon} \\
\boldsymbol{\gamma}
\end{array}\right\}
$$

where

$$
\begin{aligned}
\boldsymbol{\sigma} & =\left\{\begin{array}{c}
\sigma_{x} \\
\sigma_{y} \\
\tau_{x y}
\end{array}\right\}, \\
\boldsymbol{\tau} & =\left\{\begin{array}{c}
\tau_{x z} \\
\tau_{y z}
\end{array}\right\}, \\
\mathbf{D}_{b} & =\left[\begin{array}{ccc}
Q_{11} & Q_{12} & 0 \\
Q_{12} & Q_{22} & 0 \\
0 & 0 & Q_{66}
\end{array}\right], \\
\mathbf{D}_{s} & =\left[\begin{array}{ccc}
Q_{55} & 0 \\
0 & Q_{44}
\end{array}\right] .
\end{aligned}
$$

2.2.3. Governing Equations. The Hamilton's principle is employed to obtain the equations of motion

$$
0=\int_{0}^{T}(\delta U-\delta V) \mathrm{d} t
$$

where $\delta U$ is the variation of the strain energy and $\delta V$ is the variation of the work done by external forces and reaction forces of the elastic foundation. The variation of the strain energy is obtained as the following expression [64]:

$$
\begin{aligned}
\delta U= & \int_{A} \int_{z}\left(\sigma_{x} \delta \varepsilon_{x}+\sigma_{y} \delta \varepsilon_{y}+\tau_{x y} \delta \gamma_{x y}+\tau_{x z} \delta \gamma_{x z}+\tau_{y z} \delta \gamma_{y z}\right) \\
& \mathrm{d} A \mathrm{~d} z
\end{aligned}
$$

After integrating through the thickness of the plates, one gets the following:

$$
\delta U=\int_{A}\left[N_{x} \delta \varepsilon_{x}^{0}+N_{y} \delta \varepsilon_{y}^{0}+N_{x y} \delta \varepsilon_{x y}^{0}+M_{x} \delta \kappa_{x}^{b}+M_{y} \delta \kappa_{y}^{b}+M_{x y} \delta \kappa_{x y}^{b}++P_{x} \delta \kappa_{x}^{s}+P_{y} \delta \kappa_{y}^{s}+P_{x y} \delta \kappa_{x y}^{s}+S_{y} \delta \gamma_{y z}^{0}+S_{x} \delta \gamma_{x z}^{0}\right] \mathrm{d} A
$$

where $N_{i j}, M_{i j}, P_{i j}$ and $S_{i j}$ are the stress resultants which are calculated by

$$
\begin{aligned}
\left(N_{x}, N_{y}, N_{x y}\right) & =\int_{z}\left(\sigma_{x}, \sigma_{y}, \sigma_{x y}\right) \mathrm{d} z, \\
\left(M_{x}, M_{y}, M_{x y}\right) & =\int_{z}\left(\sigma_{x}, \sigma_{y}, \sigma_{x y}\right) z \mathrm{~d} z, \\
\left(P_{x}, P_{y}, P_{x y}\right) & =\int_{z}\left(\sigma_{x}, \sigma_{y}, \sigma_{x y}\right) f(z) \mathrm{d} z, \\
\left(S_{x}, S_{y}\right) & =\int_{z}\left(\tau_{x z}, \tau_{y z}\right) g(z) \mathrm{d} z .
\end{aligned}
$$

After integrating through the thickness of the plates and reorder in the matrix form

$$
\left\{\begin{array}{c}
\mathbf{N} \\
\mathbf{M} \\
\mathbf{P} \\
\mathbf{S}
\end{array}\right\}=\left[\begin{array}{cccc}
\mathbf{A} & \mathbf{B} & \mathbf{E} & 0 \\
\mathbf{B} & \mathbf{D} & \mathbf{F} & 0 \\
\mathbf{E} & \mathbf{F} & \mathbf{H} & 0 \\
0 & 0 & 0 & \mathbf{A s}
\end{array}\right]\left\{\begin{array}{c}
\boldsymbol{\varepsilon}^{0} \\
\boldsymbol{\kappa}^{b} \\
\boldsymbol{\kappa}^{s} \\
\boldsymbol{\gamma}^{0}
\end{array}\right\},
$$




$$
\begin{aligned}
& \mathbf{N}=\left[\begin{array}{l}
N_{x} \\
N_{y} \\
N_{x y}
\end{array}\right], \\
& \mathbf{M}=\left[\begin{array}{l}
M_{x} \\
M_{y} \\
M_{x y}
\end{array}\right], \\
& \mathbf{P}=\left[\begin{array}{c}
P_{x} \\
P_{y} \\
P_{x y}
\end{array}\right], \\
& \mathbf{S}=\left[\begin{array}{l}
S_{x} \\
S_{y}
\end{array}\right],
\end{aligned}
$$

$(\mathbf{A}, \mathbf{B}, \mathbf{E}, \mathbf{D}, \mathbf{F}, \mathbf{H})=\int_{-h / 2}^{h / 2}\left(1, z, f, z^{2}, z f, f^{2}\right) \mathbf{D}_{b} \mathrm{~d} z$

$$
\mathbf{A s}=\int_{-h / 2}^{h / 2} \mathbf{D}_{s} g^{2} \mathrm{~d} z
$$

The reaction force of the Winkler-Pasternak is calculated by $[68,76]$

$$
R_{f}=k_{w} w-k_{s} \nabla^{2} w
$$

where $\nabla^{2}=\partial^{2} / \partial x^{2}+\partial^{2} / \partial y^{2}, \quad k_{w}$ is the stiffness of the Winkler's layer, and $k_{s}$ is the stiffness of the shear layer. When $k_{s}=0$, the Winkler-Pasternak's foundation model becomes the Winkler's foundation.

The variation of the work done by external distributed force and reaction force of the elastic foundations is calculated by $[68,76]$

$$
\delta V=\int_{A}\left(q-k_{w} w+k_{s} \nabla^{2} w\right) \delta w \mathrm{~d} A .
$$

Substituting equations (18) and (25) into equation (16) and integrating by parts, the equilibrium equations of the plates are obtained as follows:

$$
\begin{aligned}
& \delta u: \frac{\partial N_{x}}{\partial x}+\frac{\partial N_{x y}}{\partial y}=0 \\
& \delta v: \frac{\partial N_{y}}{\partial y}+\frac{\partial N_{x y}}{\partial x}=0 \\
& \delta \theta: \frac{\partial^{2} P_{x}}{\partial x^{2}}+2 \frac{\partial^{2} P_{x y}}{\partial x \partial y}+\frac{\partial^{2} P_{y}}{\partial y^{2}}-\frac{\partial S_{x}}{\partial x}-\frac{\partial S_{y}}{\partial y}=0, \\
& \delta w: \frac{\partial^{2} M_{x}}{\partial x^{2}}+2 \frac{\partial^{2} M_{x y}}{\partial x \partial y}+\frac{\partial^{2} M_{y}}{\partial y^{2}}-\frac{\partial^{2} P_{x}}{\partial x^{2}}-2 \frac{\partial^{2} P_{x y}}{\partial x \partial y}-\frac{\partial^{2} P_{y}}{\partial y^{2}}+\frac{\partial S_{x}}{\partial x}+\frac{\partial S_{y}}{\partial y}+\left(q-k_{w} w+k_{s} \nabla^{2} w\right)=0 .
\end{aligned}
$$

After inserting equation (20) into equation (26), the governing equations of the plate are obtained as follows:

$$
\begin{aligned}
& \left(E_{12}+2 E_{33}-B_{12}-2 B_{33}\right) \frac{\partial^{3} w}{\partial x \partial y^{2}}+\left(E_{12}+2 E_{33}\right) \frac{\partial^{3} \theta}{\partial x \partial y^{2}}+\left(-B_{11}+E_{11}\right) \frac{\partial^{3} w}{\partial x^{3}} \\
& +E_{11} \frac{\partial^{3} \theta}{\partial x^{3}}+\left(A_{12}+A_{33}\right) \frac{\partial^{2} v}{\partial x \partial y}+A_{11} \frac{\partial^{2} u}{\partial x^{2}}+A_{33} \frac{\partial^{2} u}{\partial y^{2}}=0 \\
& \left(E_{12}+2 E_{33}-B_{12}-2 B_{33}\right) \frac{\partial^{3} w}{\partial x^{2} \partial y}+\left(E_{12}+2 E_{33}\right) \frac{\partial^{3} \theta}{\partial x^{2} \partial y}+\left(-B_{22}+E_{22}\right) \frac{\partial^{3} w}{\partial y^{3}} \\
& +E_{22} \frac{\partial^{3} \theta}{\partial y^{3}}+\left(A_{12}+A_{33}\right) \frac{\partial^{2} v}{\partial x \partial y}+A_{22} \frac{\partial^{2} v}{\partial y^{2}}+A_{33} \frac{\partial^{2} v}{\partial x^{2}}=0
\end{aligned}
$$


TABLE 1: The deflections and stresses of the square isotropic FGM plates without elastic foundation.

\begin{tabular}{|c|c|c|c|c|c|c|c|}
\hline$p$ & Method & $w^{*}$ & $\sigma_{x}^{*}$ & $\sigma_{y}^{*}$ & $\tau_{x y}^{*}$ & $\tau_{x z}^{*}$ & $\tau_{y z}^{*}$ \\
\hline \multirow{2}{*}{ Ceramic } & Zenkour [31] & 0.4665 & 2.8932 & 1.9103 & 1.2850 & 0.5114 & 0.4429 \\
\hline & Present & 0.4665 & 2.8929 & 1.9104 & 1.2844 & 0.5127 & 0.4440 \\
\hline \multirow{2}{*}{1} & Zenkour [31] & 0.9287 & 4.4745 & 2.1692 & 1.1143 & 0.5114 & 0.5446 \\
\hline & Present & 0.9287 & 4.4740 & 2.1693 & 1.1140 & 0.5127 & 0.5459 \\
\hline \multirow{2}{*}{2} & Zenkour [31] & 1.1940 & 5.2296 & 2.0338 & 0.9907 & 0.4700 & 0.5734 \\
\hline & Present & 1.1940 & 5.2289 & 2.0338 & 0.9903 & 0.4710 & 0.5746 \\
\hline \multirow{2}{*}{3} & Zenkour [31] & 1.3200 & 5.6108 & 1.8593 & 1.0047 & 0.4367 & 0.5629 \\
\hline & Present & 1.3200 & 5.6100 & 1.8594 & 1.0043 & 0.4376 & 0.5640 \\
\hline \multirow{2}{*}{4} & Zenkour [31] & 1.3890 & 5.8915 & 1.7197 & 1.0298 & 0.4204 & 0.5346 \\
\hline & Present & 1.3890 & 5.8907 & 1.7198 & 1.0293 & 0.4213 & 0.5357 \\
\hline \multirow{2}{*}{5} & Zenkour [31] & 1.4356 & 6.1504 & 1.6104 & 1.0451 & 0.4177 & 0.5031 \\
\hline & Present & 1.4356 & 6.1496 & 1.6105 & 1.0446 & 0.4185 & 0.5040 \\
\hline \multirow{2}{*}{6} & Zenkour [31] & 1.4727 & 6.4043 & 1.5214 & 1.0536 & 0.4227 & 0.4755 \\
\hline & Present & 1.4727 & 6.4034 & 1.5215 & 1.0531 & 0.4235 & 0.4764 \\
\hline \multirow{2}{*}{7} & Zenkour [31] & 1.5049 & 6.6547 & 1.4467 & 1.0589 & 0.4310 & 0.4543 \\
\hline & Present & 1.5049 & 6.6537 & 1.4468 & 1.0584 & 0.4318 & 0.4551 \\
\hline \multirow{2}{*}{8} & Zenkour [31] & 1.5343 & 6.8999 & 1.3829 & 1.0628 & 0.4399 & 0.4392 \\
\hline & Present & 1.5342 & 6.8989 & 1.3830 & 1.0622 & 0.4407 & 0.4400 \\
\hline \multirow{2}{*}{9} & Zenkour [31] & 1.5617 & 7.1383 & 1.3283 & 1.0662 & 0.4481 & 0.4291 \\
\hline & Present & 1.5616 & 7.1373 & 1.3284 & 1.0656 & 0.4490 & 0.4299 \\
\hline \multirow{2}{*}{10} & Zenkour [31] & 1.5876 & 7.3689 & 1.2820 & 1.0694 & 0.4552 & 0.4227 \\
\hline & Present & 1.5875 & 7.3679 & 1.2821 & 1.0689 & 0.4561 & 0.4235 \\
\hline \multirow[b]{2}{*}{ Metal } & Zenkour [31] & 2.5327 & 2.8932 & 1.9103 & 1.2850 & 0.5114 & 0.4429 \\
\hline & Present & 2.5326 & 2.8929 & 1.9104 & 1.2844 & 0.5127 & 0.4440 \\
\hline
\end{tabular}

$$
\begin{gathered}
\left(2 H_{12}+4 H_{33}-2 F_{12}-4 F_{33}\right) \frac{\partial^{4} w}{\partial x^{2} \partial y^{2}}+\left(2 H_{12}+4 H_{33}\right) \frac{\partial^{4} \theta}{\partial x^{2} \partial y^{2}}+ \\
\left(-F_{11}+H_{11}\right) \frac{\partial^{4} w}{\partial x^{4}}+\left(-F_{22}+H_{22}\right) \frac{\partial^{4} w}{\partial y^{4}}+H_{11} \frac{\partial^{4} \theta}{\partial x^{4}}+H_{22} \frac{\partial^{4} \theta}{\partial y^{4}}+ \\
\left(E_{12}+2 E_{33}\right) \frac{\partial^{3} u}{\partial x}+\left(E_{12}+2 E_{33}\right) \frac{\partial^{3} v}{\partial x^{2} \partial y}-A s_{22} \frac{\partial^{2} w}{\partial y^{2}}-A s_{22} \frac{\partial^{2} \theta}{\partial y^{2}}- \\
A s_{11} \frac{\partial^{2} w}{\partial x^{2}}-A s_{11} \frac{\partial^{2} \theta}{\partial x^{2}}+E_{11} \frac{\partial^{3} u}{\partial x^{3}}+E_{22} \frac{\partial^{3} v}{\partial y^{3}}=0 \\
-\left(2 H_{12}+4 H_{33}+2 D_{12}+4 D_{33}-4 F_{12}-8 F_{33}\right) \frac{\partial^{4} w}{\partial x^{2} \partial y^{2}}-\left(2 H_{12}+4 H_{33}-\right. \\
\left.2 F_{12}-4 F_{33}\right) \frac{\partial^{4} \theta}{\partial x^{2} \partial y^{2}}-\left(H_{11}+D_{11}-2 F_{11}\right) \frac{\partial^{4} w}{\partial x^{4}}-\left(D_{22}-2 F_{22}+H_{22}\right) \frac{\partial^{4} w}{\partial y^{4}}- \\
\left(-F_{11}+H_{11}\right) \frac{\partial^{4} \theta}{\partial x^{4}}-\left(-F_{22}+H_{22}\right) \frac{\partial^{4} \theta}{\partial y^{4}}-\left(E_{12}+2 E_{33}-B_{12}-2 B_{33}\right) \frac{\partial^{3} u}{\partial x}- \\
\left(E_{12}+2 E_{33}-B_{12}-2 B_{33}\right) \frac{\partial^{3} v}{\partial x^{2} \partial y}-\left(-B_{11}+E_{11}\right) \frac{\partial^{3} u}{\partial x^{3}}-\left(E_{22}-B_{22}\right) \frac{\partial^{3} v}{\partial y^{3}}- \\
\left(-A s_{11}-k_{s}\right) \frac{\partial^{2} w}{\partial x^{2}}-\left(A s_{22}-k_{s}\right) \frac{\partial^{2} w}{\partial y^{2}}+A s_{22} \frac{\partial^{2} \theta}{\partial y^{2}}+A s_{11} \frac{\partial^{2} \theta}{\partial x^{2}}+q-k_{w} w=0
\end{gathered}
$$

2.2.4. Navier's Solution. In this study, a fully simple supported FGM plate subjected to a distributed transverse load is considered. The Navier's solution technique is employed to solve the equations of motion, the unknown displacement functions of the plates are assumed as in the following formulae: 
TABLe 2: The deflections and stresses of the square FGM plates with different values of the side-to-thickness ratio.

\begin{tabular}{|c|c|c|c|c|c|c|c|}
\hline$a / h$ & $K_{w}$ & $K_{s}$ & Method & $w^{*}$ & $\sigma_{x}^{*}$ & $\tau_{x y}^{*}$ & $\tau_{x z}^{*}$ \\
\hline \multirow{8}{*}{5} & 0 & 0 & Ameur [68] & 0.3431864 & 1.0272580 & 0.3491766 & 0.2455716 \\
\hline & & & Present & 0.3431833 & 1.0272483 & 0.3491731 & 0.2455712 \\
\hline & 100 & 0 & Ameur [68] & 0.2611226 & 0.7816172 & 0.2656804 & 0.1868497 \\
\hline & & & Present & 0.2611208 & 0.7816111 & 0.2656783 & 0.1868498 \\
\hline & 0 & 10 & Ameur [68] & 0.2117976 & 0.6339728 & 0.2154944 & 0.1515546 \\
\hline & & & Present & 0.2117964 & 0.6339689 & 0.2154931 & 0.1515549 \\
\hline & 100 & 10 & Ameur [68] & 0.1773918 & 0.5309862 & 0.1804881 & 0.1269350 \\
\hline & & & Present & 0.1773910 & 0.5309832 & 0.1804871 & 0.1269354 \\
\hline \multirow{2}{*}{10} & 100 & 10 & Ameur [68] & 0.1638971 & 1.1048040 & 0.3911616 & 0.1362969 \\
\hline & & & Present & 0.1638962 & 1.1047974 & 0.3911595 & 0.1362973 \\
\hline \multirow{2}{*}{20} & 100 & 10 & Ameur [68] & 0.1602066 & 2.2330340 & 0.7988330 & 0.1388572 \\
\hline & & & Present & 0.1602057 & 2.2330216 & 0.7988292 & 0.1388576 \\
\hline \multirow{2}{*}{50} & 100 & 10 & Ameur [68] & 0.1591483 & 5.5993760 & 2.0089200 & 0.1395914 \\
\hline & & & Present & 0.1591473 & 5.5993491 & 2.0089096 & 0.1395918 \\
\hline \multirow[b]{2}{*}{100} & 100 & 10 & Ameur [68] & 0.1589961 & 11.2035900 & 4.0212420 & 0.1396969 \\
\hline & & & Present & 0.1589952 & 11.2035259 & 4.0212215 & 0.1396974 \\
\hline
\end{tabular}

TABle 3: The deflections and stresses of the square FGM plates with different values of the power-law exponent.

\begin{tabular}{|c|c|c|c|c|c|c|c|}
\hline$p$ & $K_{w}$ & $K_{s}$ & Method & $w^{*}$ & $\sigma_{x}^{*}$ & $\tau_{x y}^{*}$ & $\tau_{x z}^{*}$ \\
\hline \multirow{8}{*}{ Ceramic } & 0 & 0 & Ameur [68] & 0.2960316 & 1.9955010 & 0.7065175 & 0.2461800 \\
\hline & & & Present & 0.2960287 & 1.9954804 & 0.7065107 & 0.2461796 \\
\hline & 100 & 0 & Ameur [68] & 0.2328956 & 1.5699110 & 0.5558353 & 0.1936761 \\
\hline & & & Present & 0.2328938 & 1.5698988 & 0.5558312 & 0.1936762 \\
\hline & 0 & 10 & Ameur [68] & 0.1928403 & 1.2999050 & 0.4602384 & 0.1603661 \\
\hline & & & Present & 0.1928391 & 1.2998964 & 0.4602354 & 0.1603664 \\
\hline & 100 & 10 & Ameur [68] & 0.1638971 & 1.1048040 & 0.3911616 & 0.1362969 \\
\hline & & & Present & 0.1638962 & 1.1047974 & 0.3911595 & 0.1362973 \\
\hline \multirow{8}{*}{1} & 0 & 0 & Ameur [68] & 0.5889103 & 3.0869970 & 0.6110370 & 0.2461801 \\
\hline & & & Present & 0.5889055 & 3.0869638 & 0.6110329 & 0.2461796 \\
\hline & 100 & 0 & Ameur [68] & 0.3825844 & 2.0054610 & 0.3969590 & 0.1599303 \\
\hline & & & Present & 0.3825824 & 2.0054456 & 0.3969574 & 0.1599306 \\
\hline & 0 & 10 & Ameur [68] & 0.2852521 & 1.4952560 & 0.2959696 & 0.1192429 \\
\hline & & & Present & 0.2852509 & 1.4952473 & 0.2959689 & 0.1192432 \\
\hline & 100 & 10 & Ameur [68] & 0.2261716 & 1.1855640 & 0.2346694 & 0.0945457 \\
\hline & & & Present & 0.2261709 & 1.1855574 & 0.2346690 & 0.0945460 \\
\hline \multirow{8}{*}{5} & 0 & 0 & Ameur [68] & 0.9118358 & 4.2488300 & 0.5754613 & 0.2016656 \\
\hline & & & Present & 0.9118252 & 4.2487616 & 0.5754556 & 0.2016651 \\
\hline & 100 & 0 & Ameur [68] & 0.4969093 & 2.3154170 & 0.3136003 & 0.1098986 \\
\hline & & & Present & 0.4969062 & 2.3153954 & 0.3135990 & 0.1098990 \\
\hline & 0 & 10 & Ameur [68] & 0.3443160 & 1.6043900 & 0.2172985 & 0.0761504 \\
\hline & & & Present & 0.3443145 & 1.6043758 & 0.2172979 & 0.0761508 \\
\hline & 100 & 10 & Ameur [68] & 0.2617760 & 1.2197820 & 0.1652073 & 0.0578955 \\
\hline & & & Present & 0.2617752 & 1.2197735 & 0.1652071 & 0.0578959 \\
\hline \multirow{8}{*}{ Metal } & 0 & 0 & Ameur [68] & 1.6070280 & 1.9955010 & 0.7065178 & 0.2461801 \\
\hline & & & Present & 1.6069441 & 1.9954804 & 0.7065107 & 0.2461796 \\
\hline & 100 & 0 & Ameur [68] & 0.6501876 & 0.8073596 & 0.2858501 & 0.0996020 \\
\hline & & & Present & 0.6501739 & 0.8073528 & 0.2858497 & 0.0996041 \\
\hline & 0 & 10 & Ameur [68] & 0.4115419 & 0.5110251 & 0.1809312 & 0.0630440 \\
\hline & & & Present & 0.4115363 & 0.5110249 & 0.1809324 & 0.0630458 \\
\hline & 100 & 10 & Ameur [68] & 0.2988967 & 0.3711501 & 0.1314078 & 0.0457879 \\
\hline & & & Present & 0.2988938 & 0.3711511 & 0.1314090 & 0.0457894 \\
\hline
\end{tabular}


TABLE 4: The effects of the power-law exponent on the deflection and stresses of the plates.

\begin{tabular}{|c|c|c|c|c|c|c|c|c|}
\hline$K_{w}$ & $K_{s}$ & $p$ & $w^{*}$ & $\sigma_{x}^{*}$ & $\sigma_{y}^{*}$ & $\tau_{x y}^{*}$ & $\tau_{x z}^{*}$ & $\tau_{y z}^{*}$ \\
\hline \multirow{12}{*}{100} & \multirow{12}{*}{0} & Ceramic & 0.364386 & 2.208369 & 1.460092 & 1.038796 & 0.426826 & 0.369642 \\
\hline & & 1 & 0.594965 & 2.736732 & 1.330657 & 0.764806 & 0.371460 & 0.395564 \\
\hline & & 2 & 0.692440 & 2.857032 & 1.116230 & 0.626650 & 0.319020 & 0.389185 \\
\hline & & 3 & 0.732030 & 2.913056 & 0.971222 & 0.612666 & 0.287642 & 0.370742 \\
\hline & & 4 & 0.751997 & 2.976602 & 0.875132 & 0.615773 & 0.272492 & 0.346511 \\
\hline & & 5 & 0.764895 & 3.051605 & 0.805419 & 0.616827 & 0.267846 & 0.322594 \\
\hline & & 6 & 0.774849 & 3.132943 & 0.750606 & 0.615567 & 0.268848 & 0.302425 \\
\hline & & 7 & 0.783344 & 3.216196 & 0.705330 & 0.613308 & 0.272220 & 0.286927 \\
\hline & & 8 & 0.790969 & 3.298541 & 0.667022 & 0.610812 & 0.276128 & 0.275686 \\
\hline & & 9 & 0.797986 & 3.378347 & 0.634339 & 0.608415 & 0.279716 & 0.267830 \\
\hline & & 10 & 0.804524 & 3.454744 & 0.606471 & 0.606240 & 0.282672 & 0.262467 \\
\hline & & Metal & 0.986867 & 0.994403 & 0.661150 & 0.595750 & 0.271301 & 0.234954 \\
\hline \multirow{12}{*}{0} & \multirow{12}{*}{10} & Ceramic & 0.300884 & 1.808923 & 1.196394 & 0.871845 & 0.365029 & 0.316124 \\
\hline & & 1 & 0.441970 & 2.011672 & 0.978500 & 0.582873 & 0.291983 & 0.310930 \\
\hline & & 2 & 0.493291 & 2.016733 & 0.788144 & 0.458342 & 0.241784 & 0.294962 \\
\hline & & 3 & 0.512922 & 2.026102 & 0.675587 & 0.440051 & 0.214279 & 0.276184 \\
\hline & & 4 & 0.522575 & 2.056313 & 0.604537 & 0.437884 & 0.200941 & 0.255524 \\
\hline & & 5 & 0.528729 & 2.099420 & 0.554003 & 0.435699 & 0.196133 & 0.236222 \\
\hline & & 6 & 0.533440 & 2.148575 & 0.514607 & 0.432584 & 0.195820 & 0.220276 \\
\hline & & 7 & 0.537434 & 2.199645 & 0.482197 & 0.429174 & 0.197427 & 0.208094 \\
\hline & & 8 & 0.540999 & 2.250323 & 0.454831 & 0.425867 & 0.199545 & 0.199225 \\
\hline & & 9 & 0.544262 & 2.299377 & 0.431505 & 0.422818 & 0.201513 & 0.192950 \\
\hline & & 10 & 0.547287 & 2.346189 & 0.411616 & 0.420069 & 0.203088 & 0.188571 \\
\hline & & Metal & 0.625946 & 0.639555 & 0.424581 & 0.382653 & 0.182711 & 0.158232 \\
\hline \multirow{12}{*}{100} & \multirow{12}{*}{10} & Ceramic & 0.254115 & 1.496843 & 0.991060 & 0.758839 & 0.325433 & 0.281833 \\
\hline & & 1 & 0.346649 & 1.519772 & 0.740904 & 0.482092 & 0.251036 & 0.267326 \\
\hline & & 2 & 0.376608 & 1.471052 & 0.576833 & 0.372580 & 0.205713 & 0.250958 \\
\hline & & 3 & 0.387463 & 1.457653 & 0.488145 & 0.355217 & 0.181565 & 0.234018 \\
\hline & & 4 & 0.392635 & 1.469414 & 0.434167 & 0.352157 & 0.169877 & 0.216022 \\
\hline & & 5 & 0.395878 & 1.493847 & 0.396368 & 0.349550 & 0.165565 & 0.199406 \\
\hline & & 6 & 0.398347 & 1.523896 & 0.367099 & 0.346423 & 0.165125 & 0.185748 \\
\hline & & 7 & 0.400441 & 1.555859 & 0.343091 & 0.343192 & 0.166349 & 0.175336 \\
\hline & & 8 & 0.402313 & 1.587809 & 0.322849 & 0.340128 & 0.168029 & 0.167760 \\
\hline & & 9 & 0.404028 & 1.618756 & 0.305603 & 0.337333 & 0.169602 & 0.162395 \\
\hline & & 10 & 0.405618 & 1.648222 & 0.290894 & 0.334822 & 0.170858 & 0.158645 \\
\hline & & Metal & 0.445203 & 0.422254 & 0.281421 & 0.299906 & 0.153335 & 0.132792 \\
\hline
\end{tabular}

$$
\begin{aligned}
& u(x, y, t)=\sum_{\alpha=1}^{\infty} \sum_{\beta=1}^{\infty} U_{\alpha \beta} \cos \eta_{\alpha} x \sin \vartheta_{\beta} y, \\
& v(x, y, t)=\sum_{\alpha=1}^{\infty} \sum_{\beta=1}^{\infty} V_{\alpha \beta} \sin \eta_{\alpha} x \cos \vartheta_{\beta} y, \\
& \theta(x, y, t)=\sum_{\alpha=1}^{\infty} \sum_{\beta=1}^{\infty} \Theta_{\alpha \beta} \sin \eta_{\alpha} x \sin \vartheta_{\beta} y, \\
& w(x, y, t)=\sum_{\alpha=1}^{\infty} \sum_{\beta=1}^{\infty} W_{\alpha \beta} \sin \eta_{\alpha} x \sin \vartheta_{\beta} y,
\end{aligned}
$$

where $\eta_{\alpha}=\alpha \pi / a$ and $\vartheta_{\beta}=\beta \pi / b$.

The transverse distributed load is expanded as follows [3]:

$$
q(x, y)=\sum_{\alpha=1}^{\infty} \sum_{\beta=1}^{\infty} \Omega_{\alpha \beta} \sin \eta_{\alpha} x \sin \vartheta_{\beta} y,
$$

where $\Omega_{\alpha \beta}$ depends on the types of the load. In the case of double sinusoidal load, $\Omega_{\alpha \beta}$ are calculated as follows:

$$
\begin{cases}\Omega_{\alpha \beta}=q_{0}, & \alpha=\beta=1, \\ \Omega_{\alpha \beta}=0, & \alpha \neq 1, \beta \neq 1 .\end{cases}
$$

In the case of uniform load, $\Omega_{\alpha \beta}$ are calculated by

$$
\begin{cases}\Omega_{\alpha \beta}=\frac{16 q_{0}}{\alpha \beta \pi^{2}}, & \alpha, \beta \text { even }, \\ 0, & \alpha, \beta \text { odd. }\end{cases}
$$

Substituting equations (28) and (29) into equations (27a) $-(27 d)$, one gets

$$
\left[\begin{array}{llll}
k_{11} & k_{12} & k_{13} & k_{14} \\
& k_{22} & k_{23} & k_{24} \\
& & k_{33} & k_{34} \\
s y s & & & k_{44}
\end{array}\right]\left\{\begin{array}{c}
U_{\alpha \beta} \\
V_{\alpha \beta} \\
\Theta_{\alpha \beta} \\
W_{\alpha \beta}
\end{array}\right\}=\left\{\begin{array}{c}
0 \\
0 \\
0 \\
\Omega_{\alpha \beta}
\end{array}\right\},
$$

where 


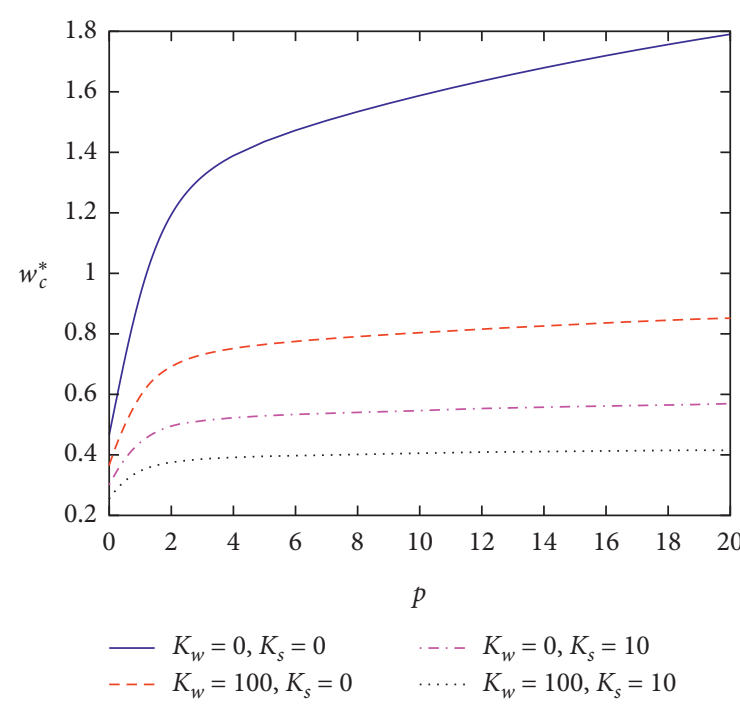

(a)

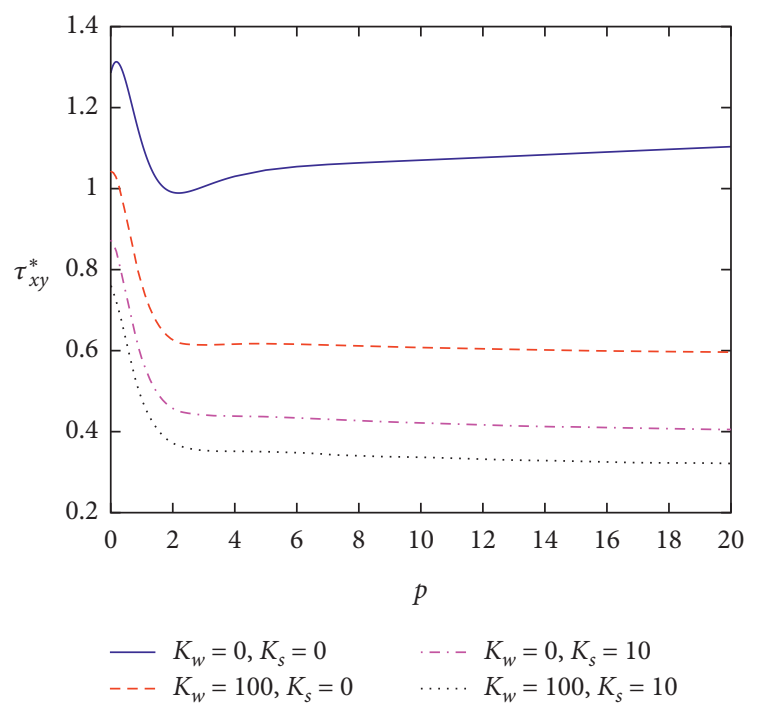

(c)

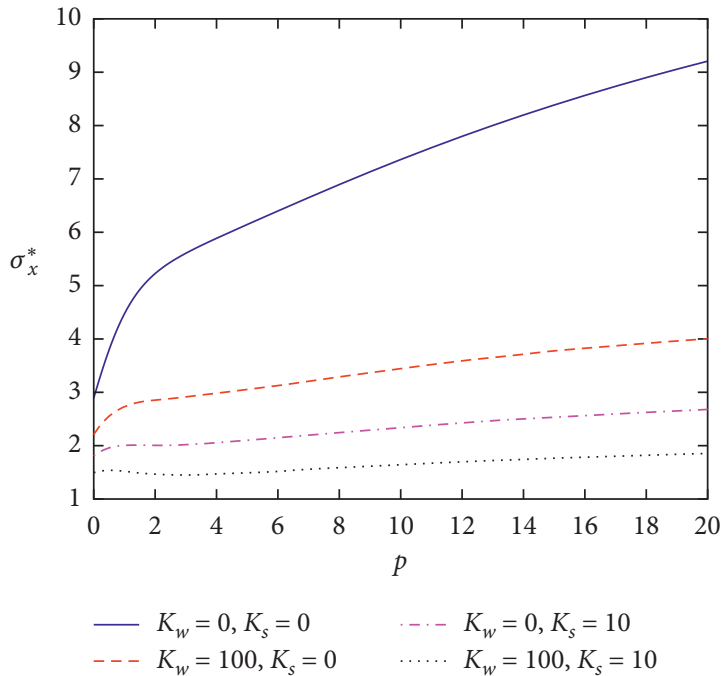

(b)

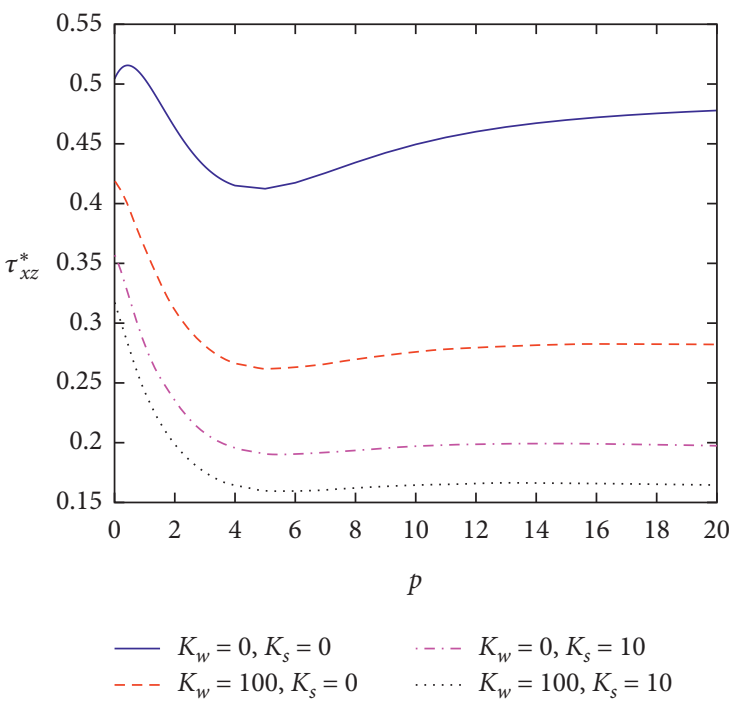

(d)

FIGURE 2: The variation of deflection and stresses as the functions of the power-law exponent.

$$
\begin{aligned}
k_{11}= & A_{11} \eta^{2}+A_{33} \vartheta^{2} ; k_{12}=\left(A_{12}+A_{33}\right) \eta \vartheta, \\
k_{13}= & E_{11} \eta^{3}+\left(E_{12}+2 E_{33}\right) \vartheta^{2} \eta, \\
k_{14}= & \left(-B_{11}+E_{11}\right) \eta^{3}+\left(E_{12}+2 E_{33}-B_{12}-2 B_{33}\right) \vartheta^{2} \eta, \\
k_{22}= & A_{22} \vartheta^{2}+A_{33} \eta^{2} ; k_{23}=E_{22} \beta^{3}+\left(E_{12}+2 E_{33}\right) \eta^{2} \vartheta, \\
k_{24}= & \left(E_{22}-B_{22}\right) \vartheta^{3}+\left(E_{12}+2 E_{33}-B_{12}-2 B_{33}\right) \eta^{2} \vartheta, \\
k_{33}= & H_{11} \eta^{4}+\left(\left(2 H_{12}+4 H_{33}\right) \vartheta^{2}+A s_{11}\right) \eta^{2}+\left(H_{22} \vartheta^{2}+A s_{22}\right) \vartheta^{2}, \\
k_{34}= & \left(-F_{11}+H_{11}\right) \eta^{4}+\left(\left(2 H_{12}+4 H_{33}-2 F_{12}-4 F_{33}\right) \vartheta^{2}+A s_{11}\right) \eta^{2}+\left(\left(H_{22}-F_{22}\right) \vartheta^{2}+A s_{22}\right) \vartheta^{2}, \\
k_{44}= & \left(-2 F_{11}+H_{11}+D_{11}\right) \eta^{4}+\left(\left(4 D_{33}-4 F_{12}-8 F_{33}+2 H_{12}+4 H_{33}+2 D_{12}\right) \vartheta^{2}+k_{s}+A s_{11}\right) \eta^{2} \\
& +\left(-2 F_{22}+H_{22}+D_{22}\right) \vartheta^{4}+\left(k_{s}+A s_{22}\right) \vartheta^{2}+k_{w} .
\end{aligned}
$$




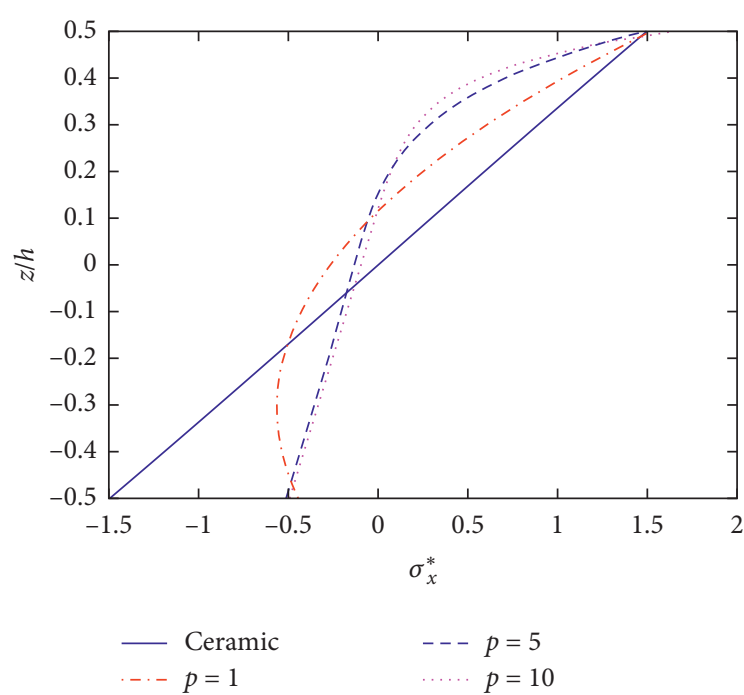

(a)

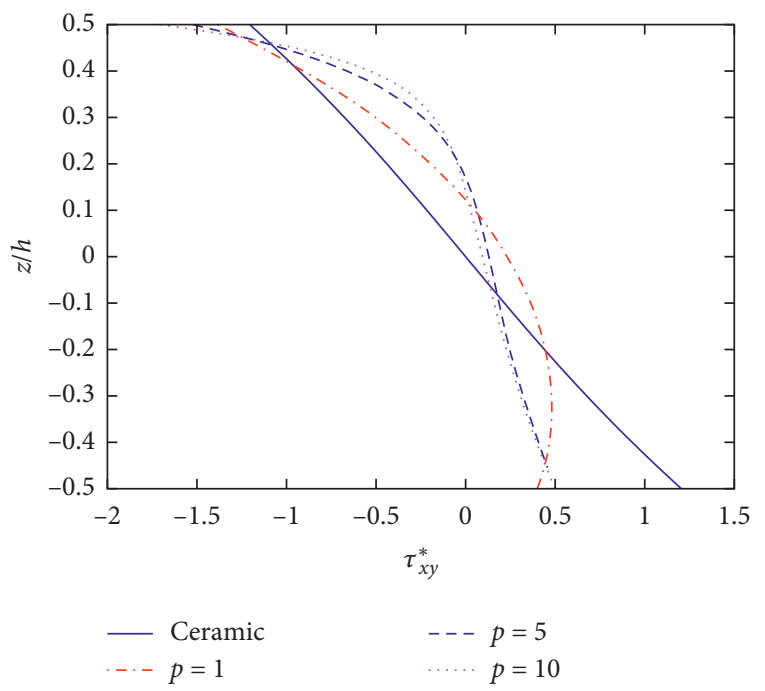

(c)

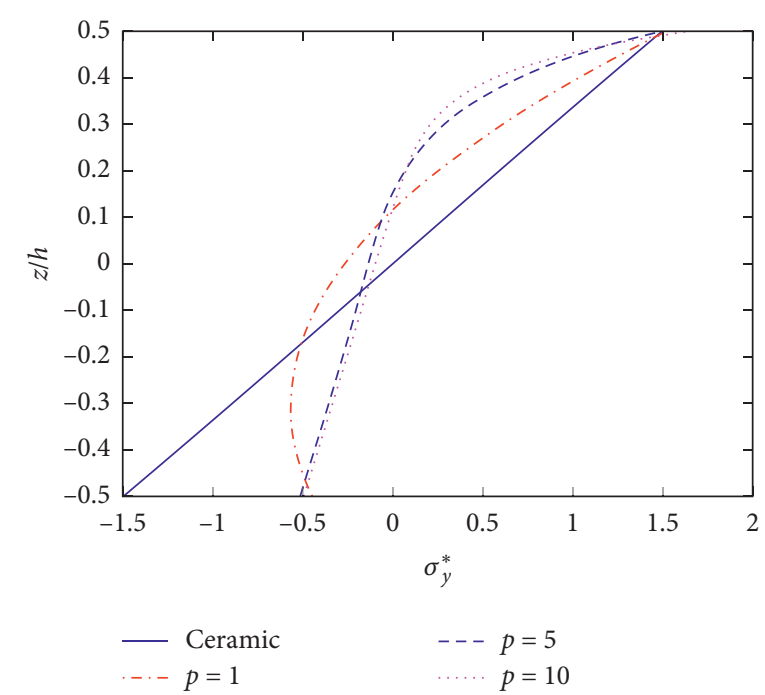

(b)

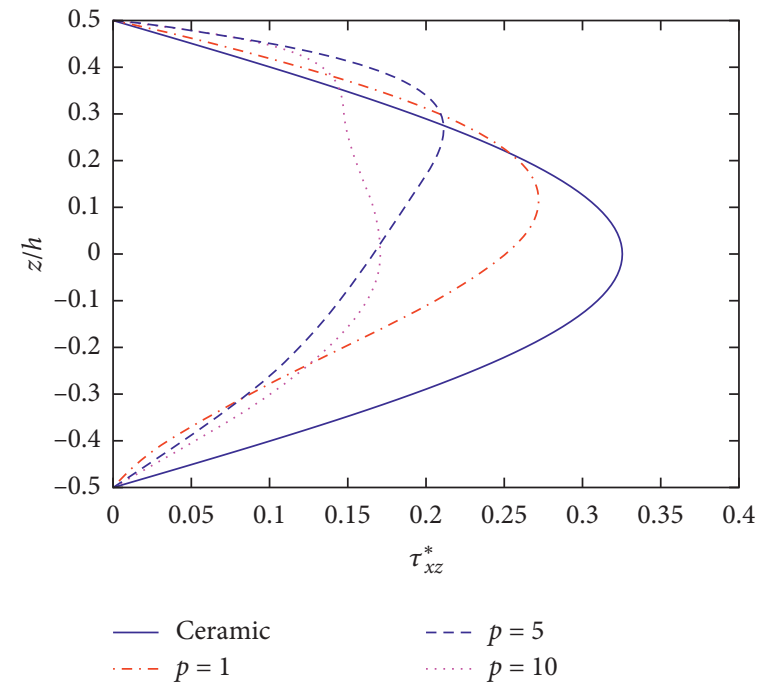

(d)

Figure 3: The distribution of the stresses through the thickness of the FGM plates.

\section{Numerical Results}

\subsection{Validation Study}

3.1.1. Static Bending of FGM Plates without Elastic Foundation. Firstly, the proposed plate theory is applied to analyze a square isotropic FGM plate of $\mathrm{Al} / \mathrm{Al}_{2} \mathrm{O}_{3}$ subjected to uniform load. In which, Young's modulus of
$\mathrm{Al}$ is $70 \mathrm{GPa}$ and it of $\mathrm{Al}_{2} \mathrm{O}_{3}$ is $380 \mathrm{GPa}$, while the Poisson's ratios are constant and equal to 0.3 , the side-tothickness ratio is $a / h=10$. The nondimensional deflection and stresses of the plates using proposed plate theory are compared with those of Zenkour [31], they are given in Table 1. The nondimensional quantities are calculated by $[31]$. 
TABLE 5: The effects of side-to-thickness ratio on the deflection and stresses of the plates.

\begin{tabular}{|c|c|c|c|c|c|c|c|c|}
\hline$K_{w}$ & $K_{s}$ & $a / h$ & $w^{*}$ & $\sigma_{x}^{*}$ & $\sigma_{y}^{*}$ & $\tau_{x y}^{*}$ & $\tau_{x z}^{*}$ & $\tau_{y z}^{*}$ \\
\hline \multirow{5}{*}{0} & \multirow{5}{*}{0} & 5 & 0.130586 & 1.143516 & 0.536764 & 0.273727 & 0.251337 & 0.267646 \\
\hline & & 10 & 0.928727 & 4.473980 & 2.169274 & 1.114001 & 0.512669 & 0.545936 \\
\hline & & 20 & 7.197403 & 17.795845 & 8.699313 & 4.478998 & 1.035194 & 1.102368 \\
\hline & & 50 & 111.442044 & 111.049014 & 54.409561 & 28.041098 & 2.601379 & 2.770184 \\
\hline & & 100 & 890.373479 & 444.096299 & 217.660390 & 112.195139 & 5.208993 & 5.547007 \\
\hline \multirow{5}{*}{100} & \multirow{5}{*}{0} & 5 & 0.120983 & 1.052603 & 0.494826 & 0.256145 & 0.237046 & 0.252428 \\
\hline & & 10 & 0.594965 & 2.736732 & 1.330657 & 0.764806 & 0.371460 & 0.395564 \\
\hline & & 20 & 1.276133 & 2.153282 & 1.059513 & 1.225915 & 0.374607 & 0.398915 \\
\hline & & 50 & 1.215731 & -0.429615 & -0.210124 & 1.892050 & 0.418211 & 0.445349 \\
\hline & & 100 & 1.220230 & 0.027689 & 0.013682 & 2.682102 & 0.497889 & 0.530197 \\
\hline \multirow{5}{*}{0} & \multirow{5}{*}{10} & 5 & 0.113048 & 0.981569 & 0.461617 & 0.240292 & 0.223360 & 0.237854 \\
\hline & & 10 & 0.441970 & 2.011672 & 0.978500 & 0.582873 & 0.291983 & 0.310930 \\
\hline & & 20 & 0.737072 & 1.488802 & 0.729602 & 0.669769 & 0.224426 & 0.238989 \\
\hline & & 50 & 0.800137 & 0.600756 & 0.294553 & 0.467580 & 0.129841 & 0.138266 \\
\hline & & 100 & 0.803947 & 0.300253 & 0.147265 & 0.305612 & 0.078395 & 0.083482 \\
\hline \multirow{5}{*}{100} & \multirow{5}{*}{10} & 5 & 0.105735 & 0.912435 & 0.429714 & 0.226881 & 0.212451 & 0.226237 \\
\hline & & 10 & 0.346649 & 1.519772 & 0.740904 & 0.482092 & 0.251036 & 0.267326 \\
\hline & & 20 & 0.492518 & 0.870810 & 0.427556 & 0.527452 & 0.194735 & 0.207371 \\
\hline & & 50 & 0.511561 & 0.320786 & 0.157370 & 0.392936 & 0.123098 & 0.131086 \\
\hline & & 100 & 0.512479 & 0.159363 & 0.078210 & 0.267070 & 0.076574 & 0.081543 \\
\hline
\end{tabular}

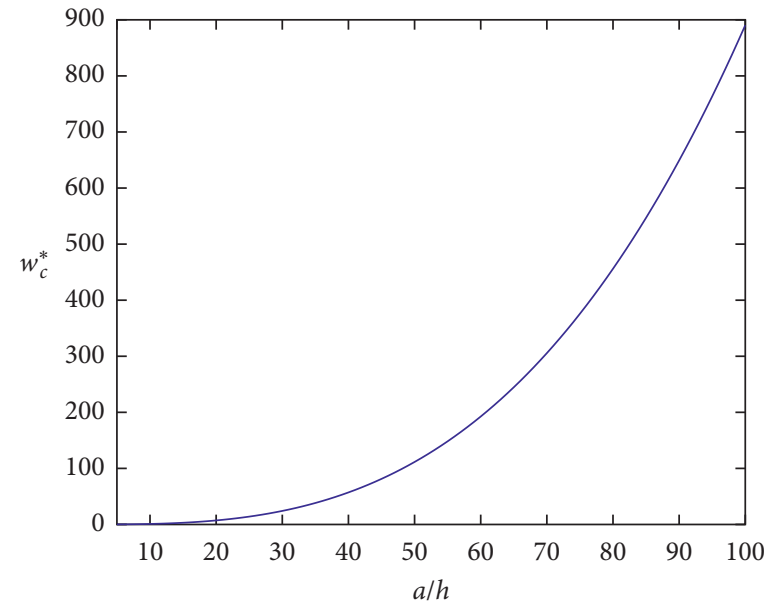

(a)

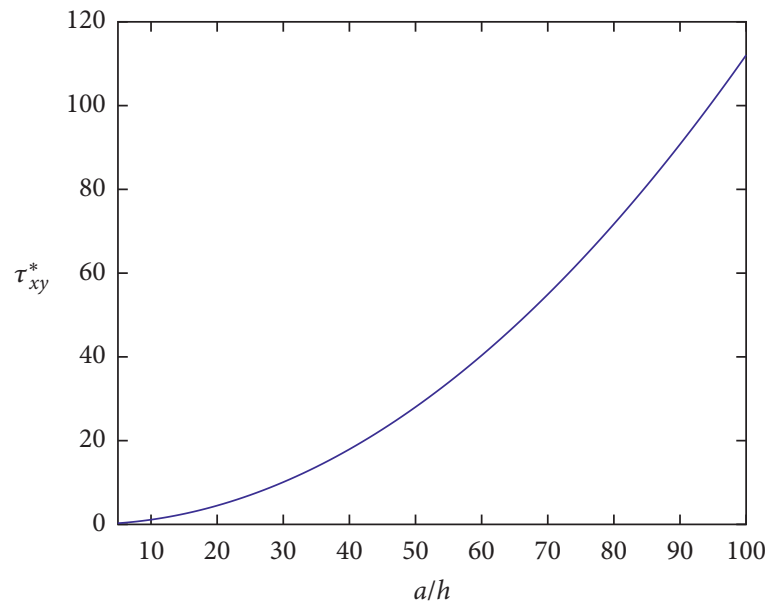

(c)

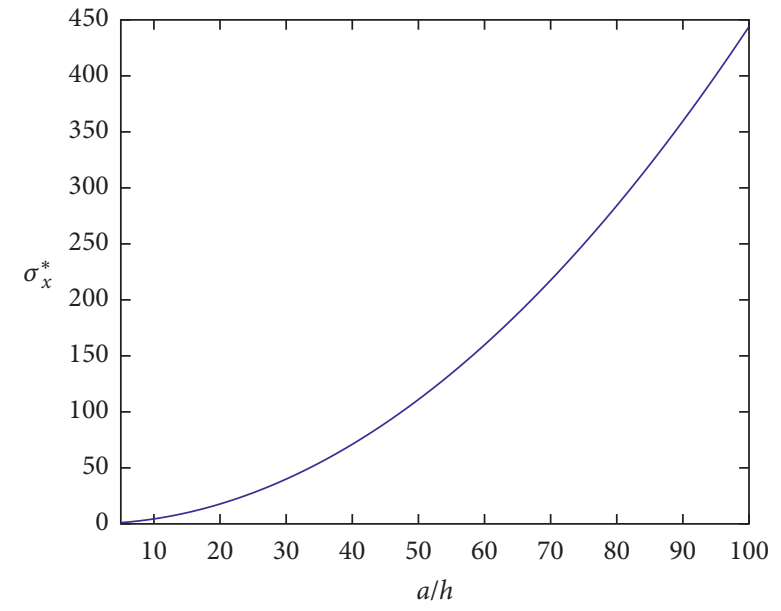

(b)

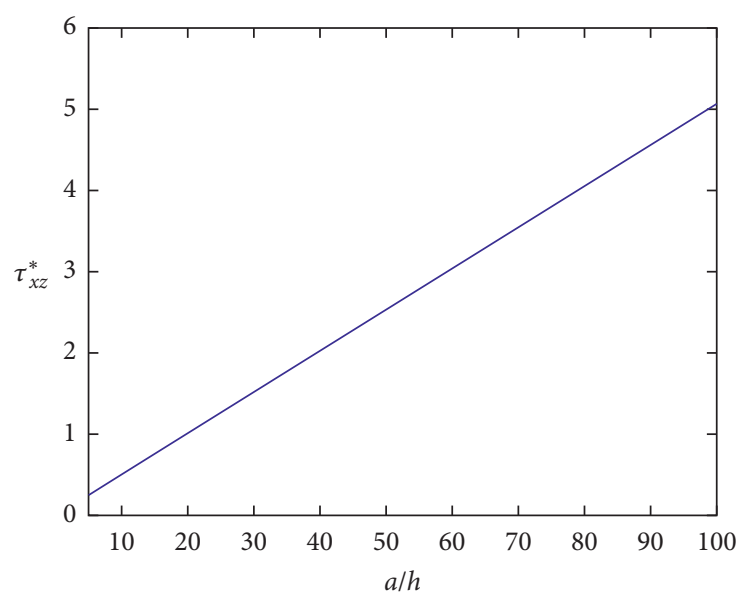

(d)

Figure 4: The variation of the deflection and stresses as the functions of side-to-thickness ratio without elastic foundation $\left(K_{w}=0, K_{s}=0\right)$. 

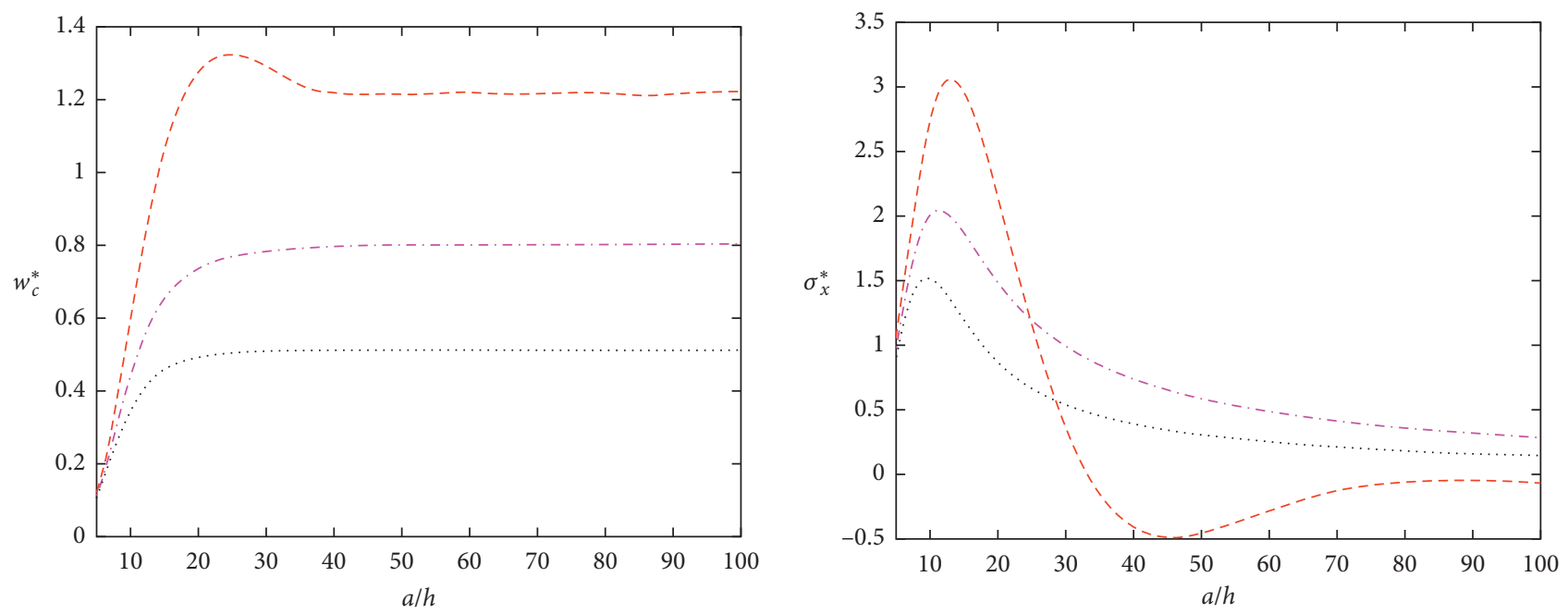

$$
\begin{array}{ll}
-- & K_{w}=100, K_{s}=0 \\
-\cdot- & K_{w}=0, K_{s}=10 \\
\cdots \cdots & K_{w}=100, K_{s}=10
\end{array}
$$

(a)

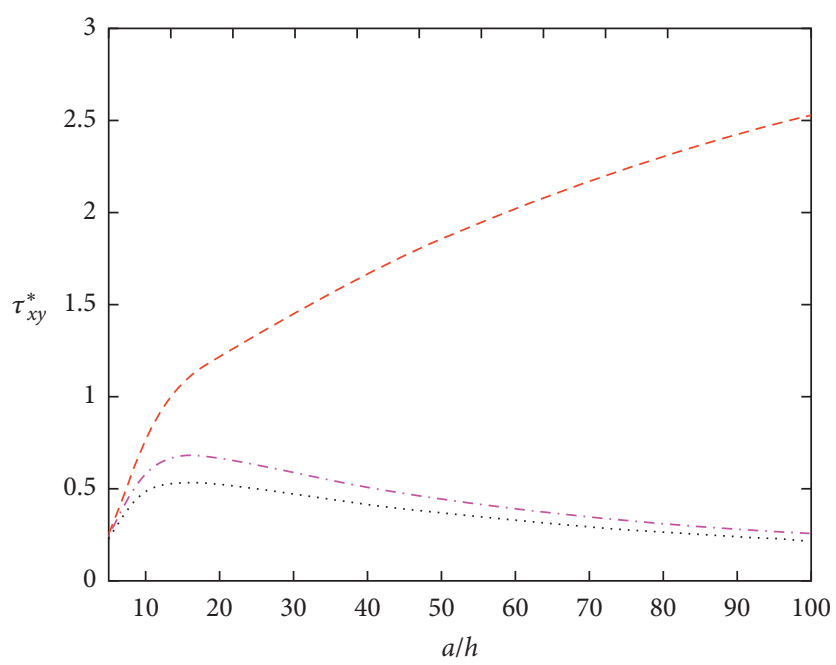

- - $K_{w}=100, K_{s}=0$

-. $K_{w}=0, K_{s}=10$

…. $K_{w}=100, K_{s}=10$

(b)

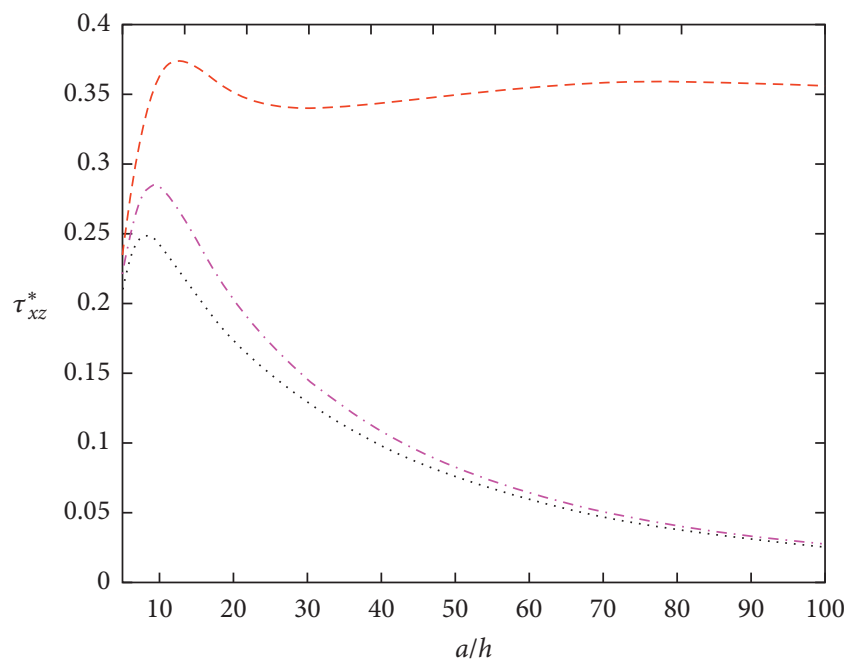

$$
\begin{array}{ll}
-- & K_{w}=100, K_{s}=0 \\
-- & K_{w}=0, K_{s}=10 \\
\cdots \cdots & K_{w}=100, K_{s}=10
\end{array}
$$

$$
\begin{array}{ll}
-- & K_{w}=100, K_{s}=0 \\
\cdots- & K_{w}=0, K_{s}=10 \\
\cdots \cdots & K_{w}=100, K_{s}=10
\end{array}
$$

(c)

(d)

Figure 5: The variation of the deflection and stresses as the functions of the side-to-thickness ratio.

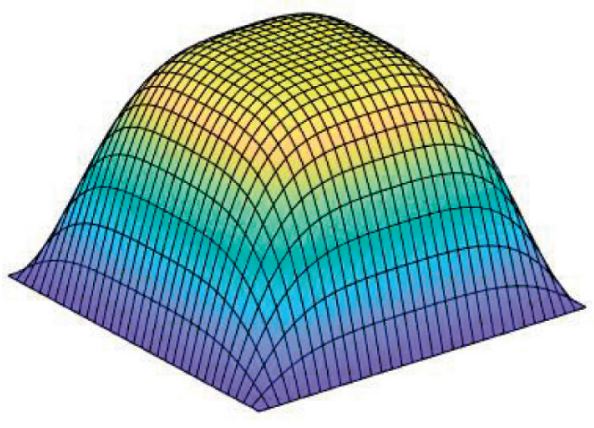

(a)

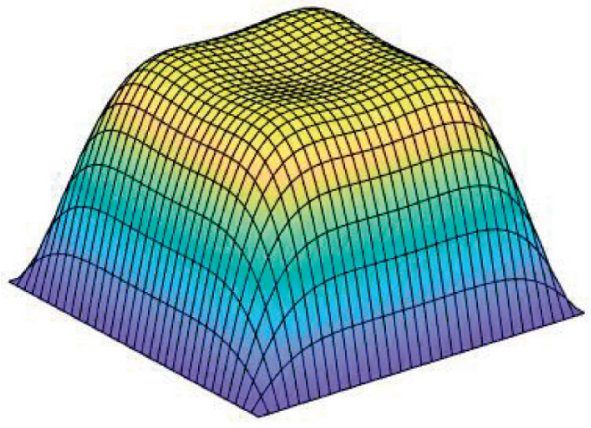

(b)

Figure 6: The deflection shapes of the plate in two case (a) $K_{w}=100, K_{s}=0, p=1, a / h=33$ and (b) $K_{w}=100, K_{s}=0, p=1, a / h=46$. 
TABLe 6: The effects of aspect ratio $a / b$ on the deflection and stresses of the plates.

\begin{tabular}{|c|c|c|c|c|c|c|c|c|}
\hline$K_{w}$ & $K_{s}$ & $a / b$ & $w^{*}$ & $\sigma_{x}^{*}$ & $\sigma_{y}^{*}$ & $\tau_{x y}^{*}$ & $\tau_{x z}^{*}$ & $\tau_{y z}^{*}$ \\
\hline \multirow{4}{*}{0} & \multirow{4}{*}{0} & 1.0 & 0.928727 & 4.473980 & 2.169274 & 1.114001 & 0.512669 & 0.545936 \\
\hline & & 1.5 & 0.357803 & 2.078198 & 1.630126 & 0.651568 & 0.365627 & 0.455065 \\
\hline & & 2.0 & 0.153645 & 1.093877 & 1.144985 & 0.392486 & 0.276125 & 0.372635 \\
\hline & & 3.0 & 0.040237 & 0.434810 & 0.589102 & 0.175661 & 0.181480 & 0.260084 \\
\hline \multirow{4}{*}{100} & \multirow{4}{*}{0} & 1.0 & 0.594965 & 2.736732 & 1.330657 & 0.764806 & 0.371460 & 0.395564 \\
\hline & & 1.5 & 0.293549 & 1.659870 & 1.322282 & 0.552956 & 0.321559 & 0.387350 \\
\hline & & 2.0 & 0.140032 & 0.982590 & 1.039558 & 0.365047 & 0.261607 & 0.344510 \\
\hline & & 3.0 & 0.039163 & 0.421653 & 0.573021 & 0.172374 & 0.179012 & 0.254131 \\
\hline \multirow{4}{*}{0} & \multirow{4}{*}{10} & 1.0 & 0.441970 & 2.011672 & 0.978500 & 0.582873 & 0.291983 & 0.310930 \\
\hline & & 1.5 & 0.210707 & 1.162850 & 0.937033 & 0.412517 & 0.250554 & 0.294494 \\
\hline & & 2.0 & 0.104255 & 0.713601 & 0.767342 & 0.283341 & 0.211786 & 0.267873 \\
\hline & & 3.0 & 0.031862 & 0.338412 & 0.464660 & 0.145296 & 0.155178 & 0.212075 \\
\hline \multirow{4}{*}{100} & \multirow{4}{*}{10} & 1.0 & 0.346649 & 1.519772 & 0.740904 & 0.482092 & 0.251036 & 0.267326 \\
\hline & & 1.5 & 0.186119 & 1.004597 & 0.819613 & 0.374334 & 0.233217 & 0.268550 \\
\hline & & 2.0 & 0.097677 & 0.660560 & 0.716501 & 0.269849 & 0.204507 & 0.254309 \\
\hline & & 3.0 & 0.031177 & 0.330115 & 0.454410 & 0.143146 & 0.153536 & 0.208284 \\
\hline
\end{tabular}

$$
\begin{aligned}
& w^{*}=\frac{10 h^{3} E_{c}}{q_{0} a^{4}} w\left(\frac{a}{2}, \frac{b}{2}\right), \\
& \sigma_{x}^{*}=\frac{h}{q_{0} a} \sigma_{x}\left(\frac{a}{2}, \frac{b}{2}, \frac{h}{2}\right), \\
& \sigma_{y}^{*}=\frac{h}{q_{0} a} \sigma_{y}\left(\frac{a}{2}, \frac{b}{2}, \frac{h}{3}\right), \\
& \tau_{x y}^{*}=\frac{h}{q_{0} a} \tau_{x y}\left(0,0,-\frac{h}{3}\right), \\
& \tau_{x z}^{*}=\frac{h}{q_{0} a} \tau_{x z}\left(0, \frac{b}{2}, 0\right), \\
& \tau_{y z}^{*}=\frac{h}{q_{0} a} \tau_{y z}\left(\frac{a}{2}, 0, \frac{h}{6}\right) .
\end{aligned}
$$

The comparison shows that the results of the proposed plate theory agree very well with those of Zenkour [31] for all cases of the power-law exponent.

3.1.2. Static Bending of FGM Plates Resting on Winkler-Pasternak Foundation. Secondly, a comparison of the deflections and stresses of the rectangular FGM plates with $p=0$ is demonstrated in Table 2. The plates are made of $\mathrm{Al} /$ $\mathrm{Al}_{2} \mathrm{O}_{3}$ with Young's modulus of $\mathrm{Al}$ is $70 \mathrm{GPa}$, and it of $\mathrm{Al}_{2} \mathrm{O}_{3}$ is $380 \mathrm{GPa}$, while the Poisson's ratios are constant and equal to 0.3 . The plates are subjected to sinusoidal load and resting on the two parameters elastic foundation. The nondimensional quantities are calculated using equation (34), while the nondimensional of two elastic foundation parameters are computed by [68]

$$
\begin{gathered}
K_{w}=\frac{a^{4}}{R} k_{w}, \\
K_{s}=\frac{a^{2}}{R} k_{s}, \\
R=\frac{E_{c} h^{3}}{12\left(1-v^{2}\right)} .
\end{gathered}
$$

In which, the numerical results of the plates using the proposed plate theory are compared with the results of Ameur [68] using a simple sinusoidal plate theory. For all cases of side-to-thickness ratio, the present numerical results are very close to those of Ameur [68].

Thirdly, the effects of the power-law exponent on the deflections and stresses of the square FGM plates are demonstrated in Table 3, in which $a / h=10$ According to Table 3, the present results are in good agreement with those of Ameur [68] for all cases of the power-law exponent and elastic foundation parameters.

3.2. Parameter Study. In this section, a rectangular FGM plate made of $\mathrm{Al} / \mathrm{Al}_{2} \mathrm{O}_{3}$ with the dimension of $a \times b$ and the thickness of $h$ resting on Winkler-Pasternak foundation is considered. The material properties of $\mathrm{Al} / \mathrm{Al}_{2} \mathrm{O}_{3}$ are similar to those of Zenkour [31] as follows

For Al: $E_{m}=70 \mathrm{GPa}, v_{m}=0.3$.

For $\mathrm{Al}_{2} \mathrm{O}_{3}: E_{c}=380 \mathrm{GPa}, v_{c}=0.3$.

The following nondimensional quantities are used for convenience [68] 

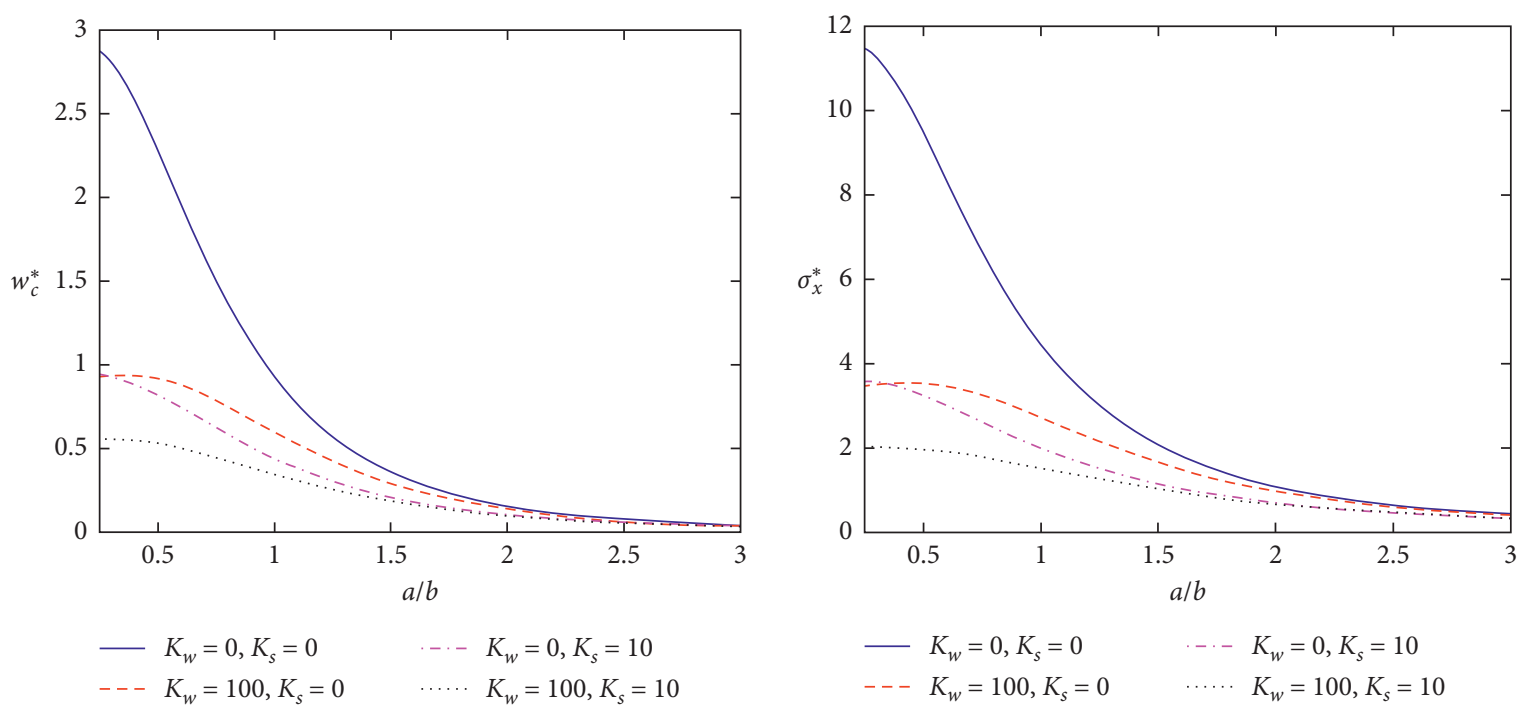

(a)

(b)
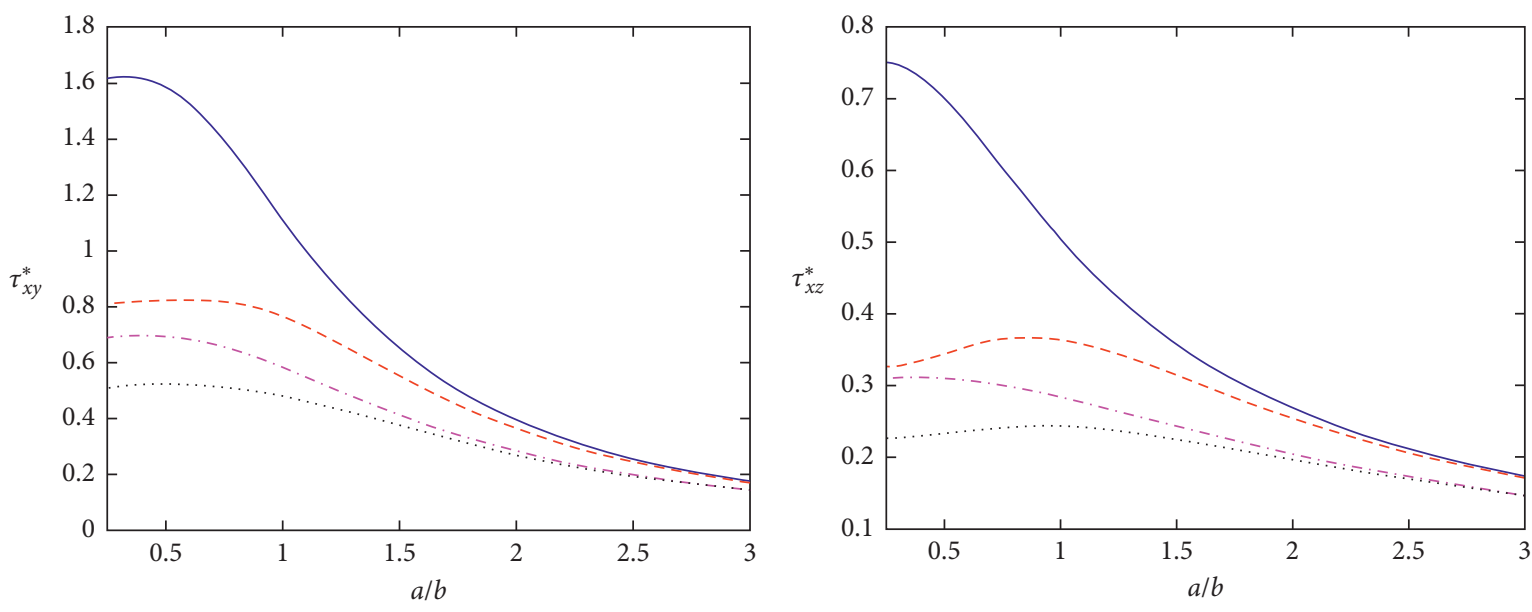

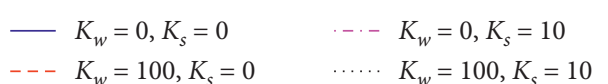

(c)

$$
\begin{aligned}
& \begin{array}{l}
-K_{w}=0, K_{s}=0 \\
-\ldots K_{w}=100, K_{s}=
\end{array} \\
& \text {-. } K_{w}=0, K_{s}=10 \\
& \text { … } K_{w}=100, K_{s}=10
\end{aligned}
$$

(d)

FIgURE 7: The variation of the deflection and stresses as the functions of aspect ratio. 
TABLE 7: The influence of two elastic foundation parameters on the deflection and stresses of the plates.

\begin{tabular}{|c|c|c|c|c|c|c|c|c|}
\hline$p$ & $K_{w}$ & $K_{s}$ & $w^{*}$ & $\sigma_{x}^{*}$ & $\sigma_{y}^{*}$ & $\tau_{x y}^{*}$ & $\tau_{x z}^{*}$ & $\tau_{y z}^{*}$ \\
\hline \multirow{9}{*}{ Ceramic } & \multirow{3}{*}{0} & 0 & 0.466544 & 2.892900 & 1.910362 & 1.284360 & 0.512669 & 0.443984 \\
\hline & & 5 & 0.366110 & 2.233322 & 1.476016 & 1.035604 & 0.423956 & 0.367156 \\
\hline & & 10 & 0.300884 & 1.808923 & 1.196394 & 0.871845 & 0.365029 & 0.316124 \\
\hline & \multirow{3}{*}{50} & 0 & 0.409357 & 2.509476 & 1.658164 & 1.146996 & 0.464665 & 0.402412 \\
\hline & & 5 & 0.329621 & 1.989165 & 1.315400 & 0.947737 & 0.393215 & 0.340534 \\
\hline & & 10 & 0.275596 & 1.640065 & 1.085299 & 0.810796 & 0.343646 & 0.297607 \\
\hline & \multirow{3}{*}{100} & 0 & 0.364386 & 2.208369 & 1.460092 & 1.038796 & 0.426826 & 0.369642 \\
\hline & & 5 & 0.299575 & 1.788415 & 1.183327 & 0.875256 & 0.367837 & 0.318557 \\
\hline & & 10 & 0.254115 & 1.496843 & 0.991060 & 0.758839 & 0.325433 & 0.281833 \\
\hline \multirow{9}{*}{0.5} & \multirow{3}{*}{0} & 0 & 0.715351 & 3.797329 & 2.123720 & 1.260322 & 0.524196 & 0.507197 \\
\hline & & 5 & 0.503375 & 2.606894 & 1.459841 & 0.924420 & 0.398993 & 0.386054 \\
\hline & & 10 & 0.387442 & 1.965189 & 1.101672 & 0.736806 & 0.328112 & 0.317472 \\
\hline & \multirow{3}{*}{50} & 0 & 0.589072 & 3.072212 & 1.719868 & 1.066647 & 0.453494 & 0.438788 \\
\hline & & 5 & 0.436582 & 2.224568 & 1.246861 & 0.821585 & 0.361388 & 0.349669 \\
\hline & & 10 & 0.346201 & 1.729868 & 0.970556 & 0.673061 & 0.304761 & 0.294878 \\
\hline & \multirow{3}{*}{100} & 0 & 0.499918 & 2.561386 & 1.435324 & 0.929555 & 0.403390 & 0.390309 \\
\hline & & 5 & 0.385028 & 1.930177 & 1.082841 & 0.741983 & 0.332242 & 0.321468 \\
\hline & & 10 & 0.312649 & 1.538904 & 0.864138 & 0.621046 & 0.285681 & 0.276417 \\
\hline \multirow{9}{*}{1.5} & \multirow{3}{*}{0} & 0 & 1.085886 & 4.927921 & 2.119488 & 1.021226 & 0.492386 & 0.566835 \\
\hline & & 5 & 0.661782 & 2.896759 & 1.248715 & 0.660779 & 0.336218 & 0.387054 \\
\hline & & 10 & 0.474174 & 2.018122 & 0.871446 & 0.496086 & 0.263344 & 0.303162 \\
\hline & \multirow{3}{*}{50} & 0 & 0.818781 & 3.616826 & 1.558387 & 0.802205 & 0.399647 & 0.460073 \\
\hline & & 5 & 0.550194 & 2.351722 & 1.015368 & 0.568731 & 0.297138 & 0.342065 \\
\hline & & 10 & 0.413220 & 1.721814 & 0.744542 & 0.445510 & 0.241815 & 0.278377 \\
\hline & \multirow{3}{*}{100} & 0 & 0.655151 & 2.816505 & 1.215781 & 0.667466 & 0.342489 & 0.394273 \\
\hline & & 5 & 0.469934 & 1.961241 & 0.848141 & 0.502217 & 0.268840 & 0.309489 \\
\hline & & 10 & 0.365692 & 1.491696 & 0.645955 & 0.405885 & 0.224911 & 0.258917 \\
\hline \multirow{9}{*}{5.5} & \multirow{3}{*}{0} & 0 & 1.454942 & 6.276915 & 1.563968 & 1.049444 & 0.420340 & 0.489465 \\
\hline & & 5 & 0.781004 & 3.222208 & 0.806585 & 0.606118 & 0.259858 & 0.302592 \\
\hline & & 10 & 0.531203 & 2.123554 & 0.533247 & 0.434216 & 0.195607 & 0.227775 \\
\hline & \multirow{3}{*}{50} & 0 & 1.010438 & 4.205625 & 1.052053 & 0.769002 & 0.321711 & 0.374616 \\
\hline & & 5 & 0.628729 & 2.517448 & 0.632253 & 0.509231 & 0.225652 & 0.262760 \\
\hline & & 10 & 0.454964 & 1.772825 & 0.446424 & 0.385332 & 0.178287 & 0.207606 \\
\hline & \multirow{3}{*}{100} & 0 & 0.770115 & 3.091798 & 0.776578 & 0.616394 & 0.267885 & 0.311938 \\
\hline & & 5 & 0.524707 & 2.038749 & 0.513753 & 0.442585 & 0.202049 & 0.235276 \\
\hline & & 10 & 0.397176 & 1.508456 & 0.380932 & 0.348025 & 0.165027 & 0.192166 \\
\hline \multirow{9}{*}{ Metal } & \multirow{3}{*}{0} & 0 & 2.532557 & 2.892900 & 1.910362 & 1.284360 & 0.512669 & 0.443984 \\
\hline & & 5 & 1.009629 & 1.074906 & 0.712260 & 0.575406 & 0.256332 & 0.221990 \\
\hline & & 10 & 0.625946 & 0.639555 & 0.424581 & 0.382653 & 0.182711 & 0.158232 \\
\hline & \multirow{3}{*}{50} & 0 & 1.431068 & 1.535900 & 1.017604 & 0.795403 & 0.341561 & 0.295800 \\
\hline & & 5 & 0.765990 & 0.778232 & 0.516957 & 0.465679 & 0.217676 & 0.188513 \\
\hline & & 10 & 0.521117 & 0.513032 & 0.341247 & 0.334892 & 0.165792 & 0.143580 \\
\hline & \multirow{3}{*}{100} & 0 & 0.986867 & 0.994403 & 0.661150 & 0.595750 & 0.271301 & 0.234954 \\
\hline & & 5 & 0.614212 & 0.595309 & 0.396460 & 0.396463 & 0.193156 & 0.167278 \\
\hline & & 10 & 0.445203 & 0.422254 & 0.281421 & 0.299906 & 0.153335 & 0.132792 \\
\hline
\end{tabular}




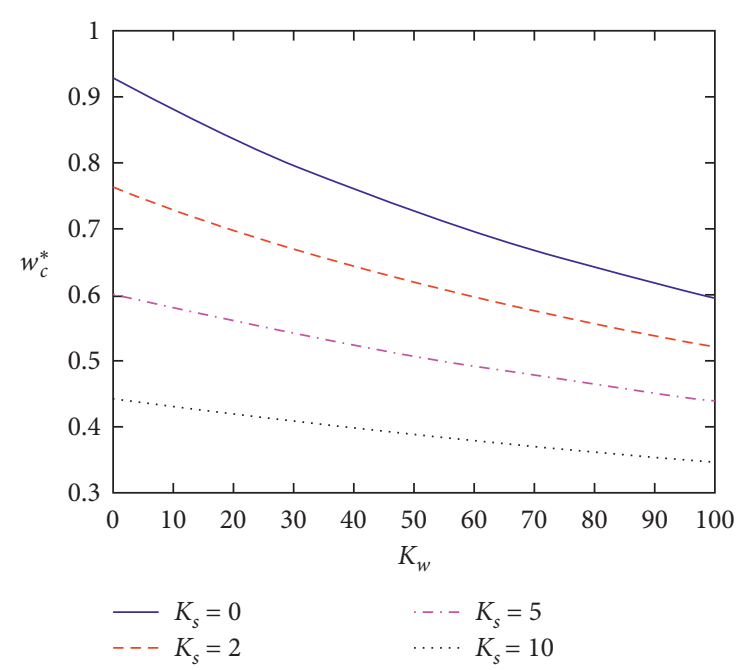

(a)

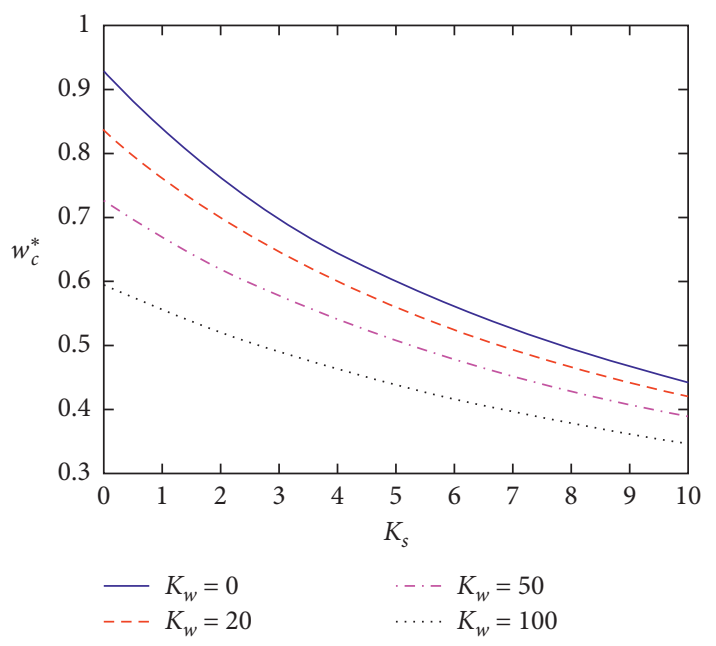

(b)

Figure 8: The influence of two parameters of the elastic foundations on the deflection of the plates with $a / h=10, p=1$.

$$
\begin{gathered}
w^{*}=\frac{10 h^{3} E_{c}}{q_{0} a^{4}} w\left(\frac{a}{2}, \frac{b}{2}\right), \\
\sigma_{x}^{*}=\frac{h}{q_{0} a} \sigma_{x}\left(\frac{a}{2}, \frac{b}{2}, \frac{h}{2}\right), \\
\sigma_{y}^{*}=\frac{h}{q_{0} a} \sigma_{y}\left(\frac{a}{2}, \frac{b}{2}, \frac{h}{3}\right), \\
\tau_{x y}^{*}=\frac{h}{q_{0} a} \tau_{x y}\left(0,0,-\frac{h}{3}\right), \\
\tau_{x z}^{*}=\frac{h}{q_{0} a} \tau_{x z}\left(0, \frac{b}{2}, 0\right), \\
\tau_{y z}^{*}=\frac{h}{q_{0} a} \tau_{y z}\left(\frac{a}{2}, 0, \frac{h}{6}\right), \\
K_{w}=\frac{a^{4}}{R} k_{w}, K_{s}=\frac{a^{2}}{R} k_{s},
\end{gathered}
$$

where $R=E_{c} h^{3} / 12\left(1-v^{2}\right)$.

3.2.1. The Effects of the Power-Law Exponent. In this subsection, the influence of the power-law exponent on the static bending behavior of the square FGM plates with $a / h=$ 10 resting on the Winkler-Pasternak foundations is investigated. The numerical results of the deflections and stresses are demonstrated in Table 4 and Figure 2. It can be seen clearly that the central deflections of the FGM plates and normal stress $\sigma_{x}^{*}, \sigma_{y}^{*}$ increase as the power-law index increase for all cases of two parameters of elastic foundations. Besides, the elastic foundations have strong effects on the bending behavior of the plates. The effects of the shear layer are stronger than the Winkler layer. However, the in-plane shear stress $\tau_{x y}^{*}$ and transverse shear stress $\tau_{x z}^{*}, \tau_{y z}^{*}$ first decrease and then increase with the increase of $p$. Especially in the case of the plate without elastic foundation, there is a small range of the power-law index in which the in-plane shear stress $\tau_{x y}^{*}$ and transverse shear stress $\tau_{x z}^{*}, \tau_{y z}^{*}$ increase when the power-law index increases, and then they decrease as shown in Figure 2.

Figure 3 displays the distribution of the stresses through the thickness of the square FGM plates resting on Winkler-Pasternak foundations with different values of the power-law index in the case of $K_{w}=100, K_{s}=10$. It is seen that the distribution of normal stresses $\sigma_{x}^{*}, \sigma_{y}^{*}$ and in-plane shear stress $\tau_{x y}^{*}$ is linear when $p=0$ and is nonlinear when $p>0$. Besides, the distribution of the transverse shear stress $\tau_{x z}^{*}$ is parabolical and symmetrical through the thickness when $p=0$, and unsymmetrical when $p>0$. In addition, the transverse shear stresses equal to zero at the top and bottom surfaces of the FGM plates.

3.2.2. The Effects of Side-to-thickness Ratio. Continuously, the effects of the side-to-thickness ratio on the bending behavior of the square FGM plate resting on the Winkler-Pasternak foundation are investigated. The power-law index of the material is $p=1$ and four cases of two parameters of Winkler-Pasternak foundations are considered. The numerical results of the effects of $a / h$ ratios are shown in Table 5, Figures 4 and 5. It is noticed that the thickness $h$ in the nondimensional formulae is fixed and equal to $a / 10$ in this subsection. As a consequence, two parameters of the Winkler-Pasternak foundations are constant as the varying of $a / h$ ratio. It is obvious that the defections and stresses of the FGM plates without elastic foundations increase rapidly when $a / h$ ratio increases as shown in Table 5 and Figure 4.

According to Figure 5, it can see that when the FGM plates are resting on elastic foundation, the central deflections of the FGM plates increase at a lower speed in comparison with the case of FGM plates without an elastic foundation. Moreover, the elastic foundations have strong 


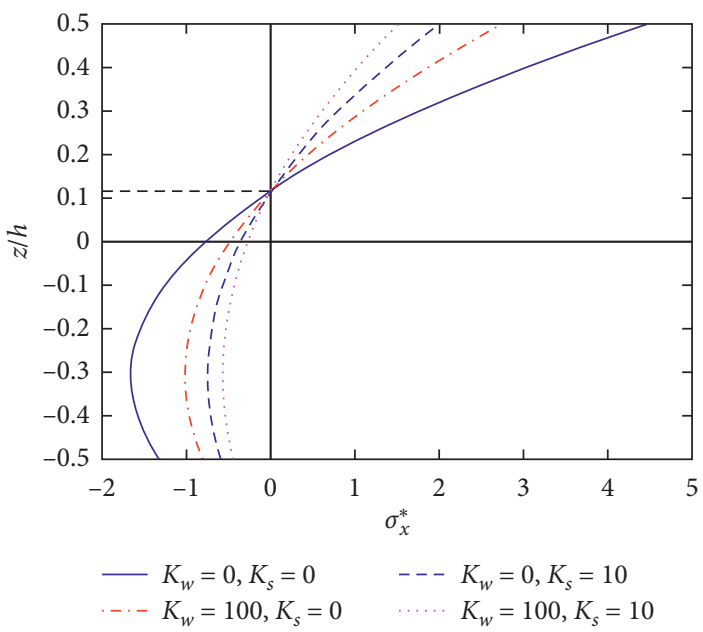

(a)

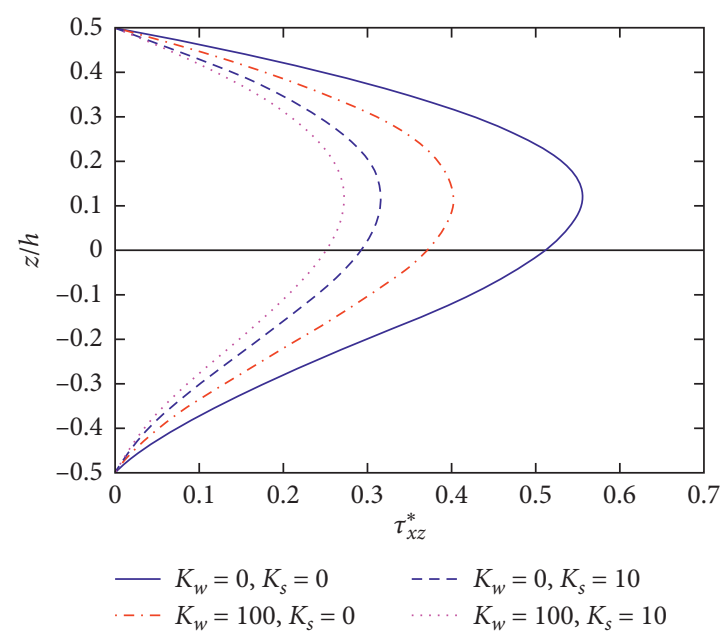

(b)

Figure 9: The influence of two parameters of the elastic foundations on the distribution of the stresses with $a / h=10, p=1$.

effects on the tresses of the FGM plates. Especially in the case of $K_{w}=100, K_{s}=0$ (Winkler type foundation), the normal stress $\sigma_{x}^{*}$ is positive when $a / h$ ratio is small and becomes negative when $a / h$ ratio is greater. It means that when the plate is thick, the deflection at the centre of the plate is local convex, and when the plate is very thin, the deflection is local concave at the centre of the plate. It is a special phenomenon of the plates resting on the elastic foundation in comparison with the plates without elastic foundation supported. In this numerical study, the normal stress $\sigma_{x}^{*}$ approximates to zero when $a / h=33$ (critical point) and the minimum normal stress $\sigma_{x}^{*}$ (compressive stress) occurs when $a / h=46$. Figure 6 shows the deflection shapes of the FGM plates in two cases of $K_{w}=100, K_{s}=$ $0, a / h=33, p=1$ and. $K_{w}=100, K_{s}=0, a / h=46, p=1$.

3.2.3. The Effects of Aspect Ratio. Table 6 and Figure 7 depict the influence of the aspect ratio on the bending behavior of the square FGM plate with $p=1$ resting on Winkler-Pasternak foundations. When the aspect ratio increases, the deflections and stresses of the FGM plates resting on the Winkler-Pasternak foundation decrease. It is obvious that the elastic foundations have significant effects on the bending behavior of the square FGM plates.

3.2.4. The Effects of the Elastic Foundations. The influences of two parameters of Winkler-Pasternak foundations on the deflection and stresses of the square FGM plates are demonstrated in Table 7, Figures 8 and 9 . The side-to-thickness ratio equals $a / h=10$. According to Table 7 and Figure 8, when the values of two parameters of Winkler-Pasternak foundations increase, the deflections and stresses of the plates decrease. Besides, the distribution of the stresses through the FGM plates' thickness also depends on the varying of two foundation parameters. Figure 9 shows that the varying of these parameters leads to the change of the values of the stresses of the plates. However, the shape of the distribution of the stresses is almost unchanged. As a consequence, the neutral surface of the FGM plate is independent of the effects of the elastic foundations.

\section{Conclusions}

In conclusion, the new sinusoidal shear deformation plate theory has been developed successfully with some advantages such as simpler, more efficient, and high accuracy in predicting the bending behavior of the FGM plates resting on Winkler-Pasternak foundations. The proposed theory consists of only four unknown variables and the transverse displacement does not separate into bending part and shear part, which means the computing cost and time can be reduced. Besides, the proposed theory can change into other plate theories easily. The numerical results show that the Winkler-Pasternak foundations have strong effects on the bending behavior of the FGM plates, and cause some special effects on the displacement and stresses of the FGM plates. These phenomena must be considered when designing, testing, and examining the FGM plates to avoid resonance.

\section{Data Availability}

The data used to support the findings of this study are included within the article.

\section{Conflicts of Interest}

The author declares that there are no conflicts of interest regarding the publication of this paper.

\section{Acknowledgments}

This work was supported by the University of Transport and Communications Foundation for Science and Technology Development (Grant no. 786/TB). 


\section{References}

[1] M. R. Barati, "A general nonlocal stress-strain gradient theory for forced vibration analysis of heterogeneous porous nanoplates," European Journal of Mechanics-A/Solids, vol. 67, pp. 215-230, 2018.

[2] M. R. Barati, M. H. Sadr, and A. M. Zenkour, "Buckling analysis of higher order graded smart piezoelectric plates with porosities resting on elastic foundation," International Journal of Mechanical Sciences, vol. 117, pp. 309-320, 2016.

[3] J. N. Reddy, "Analysis of functionally graded plates," International Journal for Numerical Methods in Engineering, vol. 47, no. 1-3, pp. 663-684, 2000.

[4] V. H. Nam, P. V. Vinh, N. V. Chinh, D. V. Thom, and T. T. Hong, "A new beam model for simulation of the mechanical behaviour of variable thickness functionally graded material beams based on modified first order shear deformation theory," Materials, vol. 12, no. 3, Article ID 404, 2019.

[5] P. V. Vinh, "Formulation of a new mixed four-node quadrilateral element for static bending analysis of variable thickness functionally graded material plates," Mathematical Problems in Engineering, vol. 2021, Article ID 6653350, 23 pages, 2021.

[6] A. S. Rezaei, A. R. Saidi, M. Abrishamdari, and M. H. P. Mohammadi, "Natural frequencies of functionally graded plates with porosities via a simple four variable plate theory: an analytical approach," Thin-Walled Structures, vol. 120, pp. 366-377, 2017.

[7] K. Swaminathan, D. T. Naveenkumar, A. M. Zenkour, and E. Carrera, "Stress, vibration and buckling analyses of FGM plates-A state-of-the-art review," Composite Structures, vol. 120, pp. 10-31, 2015.

[8] A. W. Liessa, "The free vibration of rectangular plates," Journal of Sound and Vibration, vol. 31, pp. 257-293, 1973.

[9] R. Javaheri and M. R. Eslami, "Buckling of functionally graded plates under in-plane compressive loading," Journal of Applied Mathematics and Mechanics, vol. 82, no. 4, pp. 277-283, 2002.

[10] M. Mohammadi, A. R. Saidi, and E. Jomehzadeh, "Levy solution for buckling analysis of functionally graded rectangular plates," Applied Composite Materials, vol. 17, no. 2, pp. 81-93, 2010.

[11] Y. Hu and X. Zhang, "Parametric vibrations and stability of a functionally graded plate," Mechanics Based Design of Structures and Machines, vol. 39, no. 3, pp. 367-377, 2011.

[12] L. D. Croce and P. Venini, "Finite elements for functionally graded Reissner-Mindlin plates," Computer Methods in Applied Mechanics and Engineering, vol. 193, no. 9, pp. 705-725, 2004.

[13] M. Ganapathi, T. Prakash, and N. Sundararajan, "Influence of functionally graded material on buckling of skew plates under mechanical loads," Journal of Engineering Mechanics, vol. 132, no. 8, pp. 902-905, 2006.

[14] K.-D. Kim, G. R. Lomboy, and S.-C. Han, "Geometrically non-linear analysis of functionally graded material (FGM) plates and shells using a four-node quasi-conforming shell element," Journal of Composite Materials, vol. 42, no. 5, pp. 485-511, 2008.

[15] S. Hosseini-Hashemi, H. Rokni Damavandi Taher, H. Akhavan, and M. Omidi, "Free vibration of functionally graded rectangular plates using first-order shear deformation plate theory," Applied Mathematical Modelling, vol. 34, no. 5, pp. 1276-1291, 2010.
[16] S. Hosseini-Hashemi, M. Fadaee, and S. R. Atashipour, "A new exact analytical approach for free vibration of ReissnerMindlin functionally graded rectangular plates," International Journal of Mechanical Sciences, vol. 53, no. 1, pp. 11-22, 2011.

[17] T.-K. Nguyen, K. Sab, and G. Bonnet, "First-order shear deformation plate models for functionally graded materials," Composite Structures, vol. 83, no. 1, pp. 25-36, 2008.

[18] R. P. Shimpi, H. G. Patel, and H. Arya, "New first-order shear deformation plate theories," Journal of Applied Mechanics, vol. 74, no. 3, pp. 523-533, 2007.

[19] H.-T. Thai and D.-H. Choi, "A simple first-order shear deformation theory for the bending and free vibration analysis of functionally graded plates," Composite Structures, vol. 101, pp. 332-340, 2013.

[20] H.-T. Thai, T.-K. Nguyen, T. P. Vo, and J. Lee, "Analysis of functionally graded sandwich plates using a new first-order shear deformation theory," European Journal of Mechanics A/Solids, vol. 45, pp. 211-225, 2014.

[21] H. N. Nguyen, T. T. Hong, P. V. Vinh, N. D. Quang, and D. V. Thom, "A refined simple first-order shear deformation theory for static bending and free vibration analysis of advanced composite plates," Materials, vol. 12, no. 15, Article ID 2385, 2019.

[22] T. T. Yu, S. Yin, T. Q. Bui, and S. Hirose, "A simple FSDTbased isogeometric analysis for geometrically nonlinear analysis of functionally graded plates," Finite Elements in Analysis and Design, vol. 96, pp. 1-10, 2015.

[23] M. H. Jalaei and Ö. Civalek, "On dynamic instability of magnetically embedded viscoelastic porous FG nanobeam," International Journal of Engineering Science, vol. 143, pp. 14-32, 2019.

[24] H. N. Vu, H. N. Nguyen, V. V. Pham, N. K. Dang, V. T. Do, and V. M. Phung, "A new efficient modified first-order shear model for static bending and vibration behaviors of two-layer composite plate," Advances in Civil Engineering, vol. 2019, Article ID 6814367, 17 pages, 2019.

[25] I. Senjanović, N. Vladimir, and N. Hadzic, "Modified Mindlin plate theory and shear locking-free finite element formulation," Mechanics Research Communications, vol. 55, pp. 95104, 2014.

[26] R. Javaheri and M. R. Eslami, "Thermal buckling of functionally graded plates based on higher order theory," Journal of Thermal Stresses, vol. 25, no. 7, pp. 603-625, 2002.

[27] J. Yang, K. M. Liew, and S. Kitipornchai, "Dynamic stability of laminated FGM plates based on higher-order shear deformation theory," Computational Mechanics, vol. 33, no. 4, pp. 305-315, 2004.

[28] M. Bodaghi and A. R. Saidi, "Levy-type solution for buckling analysis of thick functionally graded rectangular plates based on the higher-order shear deformation plate theory," Applied Mathematical Modelling, vol. 34, no. 11, pp. 3659-3673, 2010.

[29] A. J. M. Ferreira, R. C. Batra, C. M. C. Roque, L. F. Qian, and P. A. L. S. Martins, "Static analysis of functionally graded plates using third-order shear deformation theory and a meshless method," Composite Structures, vol. 69, no. 4, pp. 449-457, 2005.

[30] L. V. Tran, A. J. M. Ferreira, and H. Nguyen-Xuan, "Isogeometric analysis of functionally graded plates using higherorder shear deformation theory," Composites Part B: Engineering, vol. 51, pp. 368-383, 2013.

[31] A. M. Zenkour, "Generalized shear deformation theory for bending analysis of functionally graded plates," Applied Mathematical Modelling, vol. 30, no. 1, pp. 67-84, 2006. 
[32] R. P. Shimpi, "Refined plate theory and its variants," AIAA Journal, vol. 40, no. 1, pp. 137-146, 2002.

[33] D. V. Thom, P. V. Vinh, and H. N. Nguyen, "On the development of refined plate theory for static bending behavior of functionally graded plates," Mathematical Problems in Engineering, vol. 2020, Article ID 2836763, 13 pages, 2020.

[34] H.-T. Thai and S.-E. Kim, "A simple higher-order shear deformation theory for bending and free vibration analysis of functionally graded plates," Composite Structures, vol. 96, pp. 165-173, 2013.

[35] H.-T. Thai and T. P. Vo, "A new sinusoidal shear deformation theory for bending, buckling, and vibration of functionally graded plates," Applied Mathematical Modelling, vol. 37, no. 5, pp. 3269-3281, 2013.

[36] P. V. Vinh and L. Q. Huy, "Finite element analysis of functionally graded sandwich plates with porosity via a new hyperbolic shear deformation theory," Defence Technology, 2021, In press.

[37] M. Touratier, "An efficient standard plate theory," International Journal of Engineering Science, vol. 29, no. 8, pp. 901916, 1991.

[38] B. Akgöz and Ö. Civalek, "A microstructure-dependent sinusoidal plate model based on the strain gradient elasticity theory," Acta Mechanica, vol. 226, no. 7, pp. 2277-2294, 2015.

[39] I. Mechab, B. Mechab, and S. Benaissa, "Static and dynamic analysis of functionally graded plates using four-variable refined plate theory by the new function," Composites Part B: Engineering, vol. 45, no. 1, pp. 748-757, 2013.

[40] N. E. Meiche, A. Tounsi, N. Ziane, I. Mechab, and E. A. A. Bedia, "A new hyperbolic shear deformation theory for buckling and vibration of functionally graded sandwich plate"' International Journal of Mechanical Sciences, vol. 53, no. 4, pp. 237-247, 2011.

[41] A. Menasria, A. Bouhadra, A. Tounsi, A. A. Bousahla, and S. R. Mahmoud, "A new and simple HSDT for thermal stability analysis of FG sandwich plates," Steel and Composite Structures, vol. 25, no. 2, pp. 157-175, 2017.

[42] B. N. Pandya and T. Kant, "Higher-order shear deformable theories for flexure of sandwich plates-Finite element evaluations," International Journal of Solids and Structures, vol. 24, no. 12, pp. 1267-1286, 1988.

[43] M. Talha and B. N. Singh, "Static response and free vibration analysis of FGM plates using higher order shear deformation theory," Applied Mathematical Modelling, vol. 34, no. 12, pp. 3991-4011, 2010.

[44] D. V. Thom, D. K. Nguyen, N. D. Duc, D. H. Doan, and T. Q. Bui, "Analysis of bi-directional functionally graded plates by FEM and a new third-order shear deformation plate theory," Thin-Walled Structures, vol. 119, pp. 687-699, 2017.

[45] P. V. Vinh, N. T. Dung, N. C. Tho, D. V. Thom, and L. K. Hoa, "Modified single variable shear deformation plate theory for free vibration analysis of rectangular FGM plates," Structures, vol. 29, pp. 1435-1444, 2021.

[46] L. K. Hoa, P. V. Vinh, N. D. Duc, N. Thoi-Trung, L. T. Son, and D. V. Thom, "Bending and free vibration analyses of functionally graded material nanoplates via a novel nonlocal single variable shear deformation plate theory," Proceedings of the Institution of Mechanical Engineers, Part C: Journal of Mechanical Engineering Science, vol. 206, no. 1, pp. 75-77, 2019.

[47] A. M. Zenkour, "A simple four-unknown refined theory for bending analysis of functionally graded plates," Applied Mathematical Modelling, vol. 37, no. 20-21, pp. 9041-9051, 2013.
[48] V.-H. Nguyen, T.-K. Nguyen, H.-T. Thai, and T. P. Vo, “A new inverse trigonometric shear deformation theory for isotropic and functionally graded sandwich plates," Composites Part B: Engineering, vol. 66, pp. 233-246, 2014.

[49] L. F. Qian, R. C. Batra, and L. M. Chen, "Static and dynamic deformations of thick functionally graded elastic plates by using higher-order shear and normal deformable plate theory and meshless local Petrov-Galerkin method," Composites Part B: Engineering, vol. 35, no. 6-8, pp. 685-697, 2004.

[50] D. F. Gilhooley, R. C. Batra, J. R. Xiao, M. A. McCarthy, and J. W. Gillespie, "Analysis of thick functionally graded plates by using higher-order shear and normal deformable plate theory and MLPG method with radial basis functions," Composite Structures, vol. 80, no. 4, pp. 539-552, 2007.

[51] J. L. Mantari and C. Guedes Soares, "Generalized hybrid quasi-3D shear deformation theory for the static analysis of advanced composite plates," Composite Structures, vol. 94, no. 8, pp. 2561-2575, 2012.

[52] J. L. Mantari and C. Guedes Soares, "A novel higher-order shear deformation theory with stretching effect for functionally graded plates," Composites Part B: Engineering, vol. 45, no. 1, pp. 268-281, 2013.

[53] J. L. Mantari and C. Guedes Soares, "Four-unknown quasi-3D shear deformation theory for advanced composite plates," Composite Structures, vol. 109, pp. 231-239, 2014.

[54] J. L. Mantari and C. G. Soares, "A quasi-3D tangential shear deformation theory with four unknowns for functionally graded plates," Acta Mechanica, vol. 226, no. 3, pp. 625-642, 2014.

[55] H. N. Nguyen, T. T. Hong, P. V. Vinh, and D. V. Thom, "An efficient beam element based on Quasi-3D theory for static bending analysis of functionally graded beams"'” Materials, vol. 12, no. 13, Article ID 2198, 2019.

[56] A. M. Zenkour, "Benchmark trigonometric and 3-D elasticity solutions for an exponentially graded thick rectangular plate," Archive of Applied Mechanics, vol. 77, no. 4, pp. 197-214, 2007.

[57] A. M. Zenkour, "Bending analysis of functionally graded sandwich plates using a simple four-unknown shear and normal deformations theory," Journal of Sandwich Structures \& Materials, vol. 15, no. 6, pp. 629-656, 2013.

[58] P. V. Vinh, "Deflections, stresses and free vibration analysis of Bi-functionally graded sandwich plates resting on pasternak's elastic foundations via a hybrid quasi-3D theory," Mechanics Based Design of Structures and Machines, pp. 1-32, 2021, In press.

[59] H.-T. Thai and S.-E. Kim, "A simple quasi-3D sinusoidal shear deformation theory for functionally graded plates," Composite Structures, vol. 99, pp. 172-180, 2013.

[60] A. M. A. Neves, A. J. M. Ferreira, E. Carrera et al., "A quasi-3D hyperbolic shear deformation theory for the static and free vibration analysis of functionally graded plates," Composite Structures, vol. 94, no. 5, pp. 1814-1825, 2012.

[61] A. M. A. Neves, A. J. M. Ferreira, E. Carrera et al., "Static, free vibration and buckling analysis of isotropic and sandwich functionally graded plates using a quasi-3D higher-order shear deformation theory and a meshless technique," Composites Part B: Engineering, vol. 44, no. 1, pp. 657-674, 2013.

[62] S. Chakraverty and K. K. Pradhan, "Free vibration of functionally graded thin rectangular plates resting on winkler elastic foundation with general boundary conditions using Rayleigh-ritz method," International Journal of Applied Mechanics, vol. 6, no. 4, Article ID 1450043, 2014. 
[63] J. L. Mantari, E. V. Granados, and C. Guedes Soares, "Vibrational analysis of advanced composite plates resting on elastic foundation," Composites Part B: Engineering, vol. 66, pp. 407-419, 2014.

[64] S. S. Akavci, "An efficient shear deformation theory for free vibration of functionally graded thick rectangular plates on elastic foundation," Composite Structures, vol. 108, pp. 667676, 2014.

[65] H.-T. Thai, M. Park, and D.-H. Choi, "A simple refined theory for bending, buckling, and vibration of thick plates resting on elastic foundation," International Journal of Mechanical Sciences, vol. 73, pp. 40-52, 2013.

[66] H.-T. Thai and S.-E. Kim, "Closed-form solution for buckling analysis of thick functionally graded plates on elastic foundation," International Journal of Mechanical Sciences, vol. 75, pp. 34-44, 2013.

[67] A. Hasani Baferani, A. R. Saidi, and H. Ehteshami, "Accurate solution for free vibration analysis of functionally graded thick rectangular plates resting on elastic foundation," Composite Structures, vol. 93, no. 7, pp. 1842-1853, 2011.

[68] M. Ameur, A. Tounsi, I. Mechab, and A. A. El Bedia, "A new trigonometric shear deformation theory for bending analysis of functionally graded plates resting on elastic foundations," KSCE Journal of Civil Engineering, vol. 15, no. 8, pp. 14051414, 2011.

[69] S. A. Al Khateeb and A. M. Zenkour, "A refined four-unknown plate theory for advanced plates resting on elastic foundations in hygrothermal environment," Composite Structures, vol. 111, pp. 240-248, 2014.

[70] A. Attia, A. A. Bousahla, A. Tounsi, S. Mahmoud, and A. S. Alwabli, "A refined four variable plate theory for thermoelastic analysis of FGM plates resting on variable elastic foundations," Structural Engineering and Mechanics, vol. 65, no. 4, pp. 453-464, 2018.

[71] M. Avcar and W. K. M. Mohammed, "Free vibration of functionally graded beams resting on Winkler-Pasternak foundation," Arabian Journal of Geosciences, vol. 11, 2018.

[72] S. Benyoucef, I. Mechab, A. Tounsi, A. Fekrar, H. Ait Atmane, and E. A. Adda Bedia, "Bending of thick functionally graded plates resting on Winkler-Pasternak elastic foundations," Mechanics of Composite Materials, vol. 46, no. 4, pp. 425-434, 2010.

[73] J.-B. Han and K. M. Liew, "Numerical differential quadrature method for Reissner/Mindlin plates on two-parameter foundations," International Journal of Mechanical Sciences, vol. 39, no. 9, pp. 977-989, 1997.

[74] A. Gupta, M. Talha, and W. Seemann, "Free vibration and flexural response of functionally graded plates resting on Winkler-Pasternak elastic foundations using nonpolynomial higher-order shear and normal deformation theory," $M e$ chanics of Advanced Materials and Structures, vol. 25, no. 6, pp. 523-538, 2018.

[75] A. Said, M. Ameur, A. A. Bousahla, and A. Tounsi, “"A new simple hyperbolic shear deformation theory for functionally graded plates resting on Winkler-Pasternak elastic foundations"' International Journal of Computational Methods, vol. 11, no. 6, Article ID 1350098, 2014.

[76] F. Z. Zaoui, D. Ouinas, and A. Tounsi, "New 2D and quasi-3D shear deformation theories for free vibration of functionally graded plates on elastic foundations," Composites Part B: Engineering, vol. 159, pp. 231-247, 2019. 